\title{
Analysis of the semileptonic and nonleptonic two-body decays of the double heavy charm baryon states $\Xi_{c c}^{++}, \Xi_{c c}^{+}$and $\Omega_{c c}^{+}$
}

\author{
Thomas Gutsche, ${ }^{1}$ Mikhail A. Ivanov, ${ }^{2}$ Jürgen G. Körner, ${ }^{3}$ Valery E. Lyubovitskij, ${ }^{1,4,5}$ and Zhomart Tyulemissov ${ }^{2,6,7}$ \\ ${ }^{1}$ Institut für Theoretische Physik, Universität Tübingen, Kepler Center for Astro and Particle Physics, \\ Auf der Morgenstelle 14, D-72076 Tübingen, Germany \\ ${ }^{2}$ Bogoliubov Laboratory of Theoretical Physics, Joint Institute for Nuclear Research, \\ 141980 Dubna, Russia \\ ${ }^{3}$ PRISMA Cluster of Excellence, Institut für Physik, Johannes Gutenberg-Universität, \\ D-55099 Mainz, Germany \\ ${ }^{4}$ Departamento de Física y Centro Científico Tecnológico de Valparaíso-CCTVal, Universidad Técnica \\ Federico Santa María, Casilla 110-V, Valparaíso, Chile \\ ${ }^{5}$ Department of Physics, Tomsk State University, 634050 Tomsk, Russia \\ ${ }^{6}$ The Institute of Nuclear Physics, Ministry of Energy of the Republic of Kazakhstan, \\ 050032 Almaty, Kazakhstan \\ ${ }^{7}$ Al-Farabi Kazakh National University, 050038 Almaty, Kazakhstan
}

(Received 26 November 2019; published 23 December 2019)

\begin{abstract}
We calculate the semileptonic and a subclass of sixteen nonleptonic two-body decays of the double charm baryon ground states $\Xi_{c c}^{++}, \Xi_{c c}^{+}$and $\Omega_{c c}^{+}$where we concentrate on the nonleptonic decay modes. We identify those nonleptonic decay channels in which the decay proceeds solely via the factorizing contribution precluding a contamination from $W$ exchange. We use the covariant confined quark model previously developed by us to calculate the various helicity amplitudes which describe the dynamics of the $1 / 2^{+} \rightarrow 1 / 2^{+}$and $1 / 2^{+} \rightarrow 3 / 2^{+}$transitions induced by the Cabibbo-favored effective $(c \rightarrow s)$ and $(d \rightarrow u)$ currents. We then proceed to calculate the rates of the decays as well as polarization effects and angular decay distributions of the prominent decay chains resulting from the nonleptonic decays of the double heavy charm baryon parent states.
\end{abstract}

DOI: 10.1103/PhysRevD.100.114037

\section{INTRODUCTION}

Two years ago the LHCb Collaboration reported on the discovery of the double heavy charm baryon state $\Xi_{c c}^{++}[1]$. The state was found in the invariant mass spectrum of the final state particles $\left(\Lambda_{c}^{+} K^{-} \pi^{+} \pi^{+}\right)$where the $\Lambda_{c}^{+}$baryon was reconstructed in the decay mode $p K^{-} \pi^{+}$. The mass of the new state was given as $3621.40 \pm 0.72 \pm 0.14 \pm$ $0.27 \mathrm{MeV}$. A year later the $\mathrm{LHCb}$ Collaboration identified the same state in the decay $\Xi_{c c}^{++} \rightarrow \Xi_{c}^{+}+\pi^{+}$with a mass value of $3620.6 \pm 1.5$ (stat) \pm 0.4 (syst) $\pm 0.3\left(\Xi_{c}^{+}\right) \mathrm{MeV}$ [2]. The lifetime of the $\Xi_{c c}^{++}$was measured to be $\tau\left(\Xi_{c c}^{++}\right)=$ $0.256_{-0.022}^{+0.024}$ (stat) \pm 0.014 (syst) ps [3].

The weighted average of the two mass measurements of $m_{\Xi_{c c}^{++}}=3621 \pm 1.1 \pm 0.3 \pm 0.3 \mathrm{MeV}$ is quite close to the value of $3610 \mathrm{MeV}$ predicted some time ago in Ref. [4] in

Published by the American Physical Society under the terms of the Creative Commons Attribution 4.0 International license. Further distribution of this work must maintain attribution to the author(s) and the published article's title, journal citation, and DOI. Funded by SCOAP. the framework of the one-gluon exchange model of de Rujula et al. [5] with a Breit-Fermi spin-spin interaction term. It is noteworthy that Ebert et al. predicted a mass of $3620 \mathrm{MeV}$ for the $\Xi_{c c}^{++}$using a relativistic quark-diquark potential model [6]. In Ref. [7] we have interpreted the new double charm baryon state found in the $\left(\Lambda_{c}^{+} K^{-} \pi^{+} \pi^{+}\right)$mass distribution as being at the origin of the decay chain $\Xi_{c c}^{++} \rightarrow \Sigma_{c}^{++}\left(2455 ; 1 / 2^{+}\right)\left(\rightarrow \Lambda_{c}^{+} \pi^{+}\right)+\bar{K}^{* 0}\left(\rightarrow K^{-} \pi^{+}\right)$. In the present paper we extend the analysis of Ref. [7] in two directions. First, we consider the possibility that the first step in the decay chain consists of the decay $\Xi_{c c}^{++} \rightarrow$ $\Sigma_{c}^{++}\left(2520 ; 3 / 2^{+}\right)+\bar{K}^{* 0}$ where the state $\Sigma_{c}^{++}\left(2520 ; 3 / 2^{+}\right)$ is the spin $3 / 2$ heavy quark symmetry partner of the $\Sigma_{c}^{++}\left(2455 ; 1 / 2^{+}\right)$. In fact, in a talk at a CERN Seminar [8] Zhang (LHCb Collaboration) showed an invariant mass plot for the $\left(\Lambda_{c} \pi^{+}\right)$subsystem in which the peaking bin for $m\left(\Lambda_{c} \pi^{+}\right)$lies in between the two $\Sigma_{c}^{++}\left(2455 ; 1 / 2^{+}\right)$and $\Sigma_{c}^{++}\left(2520 ; 3 / 2^{+}\right)$states. Second, we provide results for a subclass of the Cabibbo-favored nonleptonic two-body decays of the not yet identified $J^{P}=1 / 2^{+}$double charm baryon ground states $\Xi_{c c}^{+}(3610)$ and $\Omega_{c c}^{+}(3710)$ where the mass values are again taken from the calculation of [4]. 
The authors of [6] predict a mass value of $M_{\Omega_{c c}^{+}}=$ $3778 \mathrm{MeV}$, which is considerably higher than the value $M_{\Omega_{c c}^{+}}=3710$ calculated in Ref. [4]. A recent lattice calculation quotes a value of $3712 \pm 10 \pm 12 \mathrm{MeV}$ for the $\Omega_{c c}^{+}$state [9].

The physics of double heavy charm and bottom baryons (mass spectrum and decay properties) has been studied before in a number of papers [4-7,9-34]. We presented a detailed analysis of exclusive decays of double heavy baryons using several versions of covariant quark models in Refs. $[7,10,12]$. Double heavy baryon decays and their magnetic moments were treated by us in Refs. [10] where we performed a comprehensive study of the semileptonic and radiative decays of double heavy baryons using a covariant quark model without implementing quark confinement. The version of the covariant quark model used in [10] has been improved by incorporating quark confinement in an effective way [35]. For the calculation of the relevant $1 / 2^{+} \rightarrow 1 / 2^{+}$and $1 / 2^{+} \rightarrow 3 / 2^{+}$transitions performed in this paper we use the improved quark model which we refer to as the covariant confined quark model (CCQM). In Refs. [7,11,12] we studied decay properties of double heavy baryons in the CCQM approach. In particular, in Ref. [7] we interpreted the $\Xi_{c c}^{++}$baryon found by the $\mathrm{LHCb}$ Collaboration in the invariant mass distribution of the set of final state particles $\left(\Lambda_{c}^{+} K \pi^{+} \pi^{+}\right)$as being at the origin of the decay chain $\Xi_{c c}^{++} \rightarrow \Sigma_{c}^{++}\left(\rightarrow \Lambda_{c}^{+} \pi^{+}\right)+$ $\bar{K}^{* 0}\left(\rightarrow K^{-} \pi^{+}\right)$. The nonleptonic decay $\Xi_{c c}^{++} \rightarrow \Sigma_{c}^{++} \bar{K}^{* 0}$ belongs to the class of factorizing decays, i.e., the decays precluding a contamination from internal $W$ exchange. As a byproduct of our investigation we have also analyzed the nonleptonic mode with $\bar{K}^{0}$ in the final state. In Ref. [11] we proposed a novel method for the evaluation of the nonfactorizing (three quark loop) diagrams generated by $W$ exchange and contributing to the nonleptonic two-body decays of the doubly charmed baryons $\Xi_{c c}^{++}$and $\Omega_{c c}^{+}$. The $W$-exchange contributions appear in addition to the factorizable tree graph contributions and are not suppressed in general. In Ref. [12] we reviewed novel ideas in the theoretical description of nonleptonic decays of double heavy baryons. In the present paper we extend our analysis of semileptonic decays of double charm baryons started in Ref. [10] by inclusion of all factorizable modes for both types of weak transitionssemileptonic and nonleptonic using the updated theoretical framework-CCQM model. Note that in our paper Ref. [10] we used mass values for the single and double charm baryons masses $\Xi_{c c}^{++}=3.61 \mathrm{GeV}$ and $\Xi_{c}^{\prime+}=$ $2.47 \mathrm{GeV}$, which differ from the updated mass values used in the present paper $\left(\Xi_{c c}^{++}=3.6206 \mathrm{GeV}\right.$ and $\Xi_{c}^{\prime+}=$ $2.5774 \mathrm{GeV}$ [36]). When comparing the relevant semileptonic rate in [10] to that in the present paper one has to take the changed mass values into account, which results in a suppression of our 2001 result by a factor of $\sim 1.6$ which is mostly kinematical in nature.
Our paper is structured as follows. In Sec. II we discuss the decay topologies of the Cabibbo-favored nonleptonic two-body decays of the double charm baryon ground states $\Xi_{c c}^{++}, \Xi_{c c}^{+}$and $\Omega_{c c}^{+}$. Of the many possible decays we identify 16 decays which proceed via the factorizing contributions alone. In Sec. III we collect material on the spin kinematics of the decays. We define invariant form factors and helicity amplitudes. We also write down formulas for the semileptonic and nonleptonic rates. In Sec. IV we list a set of local interpolating three-quark currents with the correct quantum numbers of the baryon states that they describe. The nonlocal versions of the interpolating currents enter the calculation of the various transition form factors in our CCQM. We also give a brief description of the main features of our CCQM calculation. Section V contains our numerical results for the semileptonic and nonleptonic rates and branching fractions. In Sec. V we also discuss polarization effects and angular decay distributions of the eight semileptonic and 16 nonleptonic cascade decays. In Sec. VI we summarize our results and outline our follow-up program of further calculations involving also $W$-exchange contributions to the nonleptonic decays of double heavy charm baryons.

\section{DECAY TOPOLOGIES OF CABIBBO-FAVORED DOUBLE HEAVY CHARM BARYON NONLEPTONIC DECAYS}

We begin by a discussion of the different color-flavor topologies that contribute to the various possible nonleptonic two-body transitions of the double heavy $\Xi_{c c}^{++}, \Xi_{c c}^{+}$ and $\Omega_{c c}^{+}$states. The relevant topologies are displayed in Fig. 1. We refer to the topologies of Ia and $\mathrm{Ib}$ as tree diagrams. They are sometimes also referred to as external (Ia) and internal $W$-emission (Ib) diagrams. The topologies IIa, IIb and III are referred to as $W$-exchange diagrams. In [37] they are denoted as the exchange (IIa), colorcommensurate (IIb) and bow tie (III) diagram. As shown in Fig. 1 the color-flavor factor of the tree diagrams Ia and Ib depends on whether the emitted meson is charged or neutral. For charged emission the color-flavor factor is given by the linear combination of Wilson coefficients $\left(C_{2}+\xi C_{1}\right)$, where $\xi=1 / N_{c}$, while for neutral emission the color-flavor factor reads $\left(C_{1}+\xi C_{2}\right)$. We take $C_{1}=$ -0.51 and $C_{2}=1.20$ from Ref. [38]. We use the large $N_{c}$ limit for the color-flavor factors. For the $W$-exchange diagrams not treated in this paper the color-flavor factor is given by $\left(C_{2}-C_{1}\right)$.

In Table I we provide a complete list of the Cabibbofavored ground-state to ground-state nonleptonic two-body decays of double heavy charm baryons together with the color-flavor topologies that contribute to these decays. For reasons of compactness we employ a star notation for the spin $3 / 2$ ground state baryons which differs from the notation suggested by the Particle Data Group (PDG). Thus, for example, our $\Omega_{c}^{* 0}$ stands for the spin 3/2 partner 


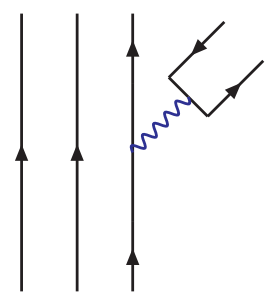

Ia

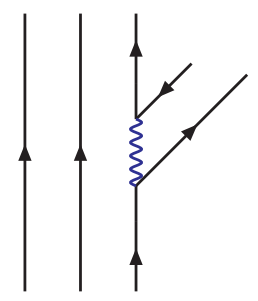

$\mathrm{Ib}$

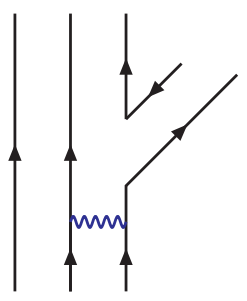

IIa

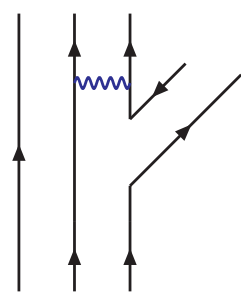

IIb

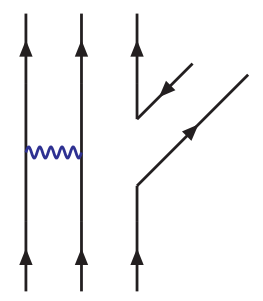

III

charged meson: $C_{2}+\xi C_{1}$

$C_{2}-C_{1}$

$C_{2}-C_{1}$

$C_{2}-C_{1}$

FIG. 1. Flavor-color topologies of nonleptonic weak decays.

of the spin $1 / 2$ state $\Omega_{c}^{0}$. The spin $3 / 2$ state $\Omega_{c}^{* 0}$ is listed in the PDG [36] as $\Omega_{c}(2770)^{0}$.

In this paper we restrict our analysis to those nonleptonic decays whose decay dynamics is solely determined by the tree diagram contributions Ia and Ib. There are two classes of such decays which we discuss in turn.

(i) The first class of decays is solely contributed to by the two topologies Ia and Ib. These decays can be identified by necessary and sufficient conditions for the quarks involved in the two-body nonleptonic transitions which we label according to the following scheme:

$$
B_{1}\left(q_{1} q_{2} q_{3}\right) \rightarrow B_{2}\left(q_{1}^{\prime} q_{2}^{\prime} q_{3}^{\prime}\right)+M\left(q_{m} \bar{q}_{\bar{n}}\right) .
$$

A necessary condition for the contribution of the factorizing class of decays is that a quark pair

TABLE I. Cabibbo-favored nonleptonic two-body decays of double heavy charm baryons including $W$-exchange contributions.

\begin{tabular}{lccccc}
\hline \hline & $I_{a}$ & $I_{b}$ & $I_{a}$ & $I_{b}$ & $I I I$ \\
\hline$\Xi_{c c}^{++} \rightarrow \Sigma_{c}^{(*)++}+\bar{K}^{(*) 0}$ & $\ldots$ & $\checkmark$ & $\ldots$ & $\ldots$ & $\ldots$ \\
$\Xi_{c c}^{++} \rightarrow \Xi_{c}^{(\prime, *)+}+\pi^{+}\left(\rho^{+}\right)$ & $\checkmark$ & $\ldots$ & $\ldots$ & $\checkmark$ & $\ldots$ \\
$\Xi_{c c}^{++} \rightarrow \Sigma^{(*)+}+D^{(*)+}$ & $\ldots$ & $\ldots$ & $\ldots$ & $\checkmark$ & $\ldots$ \\
$\Xi_{c c}^{+} \rightarrow \Xi_{c}^{(\prime, *) 0}+\pi^{+}\left(\rho^{+}\right)$ & $\checkmark$ & $\ldots$ & $\checkmark$ & $\ldots$ & $\ldots$ \\
$\Xi_{c c}^{+} \rightarrow \Lambda_{c}^{+}\left(\Sigma_{c}^{(*)+}\right)+\bar{K}^{(*) 0}$ & $\ldots$ & $\checkmark$ & $\checkmark$ & $\ldots$ & $\ldots$ \\
$\Xi_{c c}^{+} \rightarrow \Sigma_{c}^{(*)++}+K^{(*)-}$ & $\ldots$ & $\ldots$ & $\checkmark$ & $\ldots$ & $\ldots$ \\
$\Xi_{c c}^{+} \rightarrow \Xi_{c}^{(\prime, *)+}+\pi^{0}\left(\rho^{0}\right)$ & $\ldots$ & $\ldots$ & $\checkmark$ & $\checkmark$ & $\ldots$ \\
$\Xi_{c c}^{+} \rightarrow \Xi_{c}^{(\prime, *)+}+\eta\left(\eta^{\prime}\right)$ & $\ldots$ & $\ldots$ & $\checkmark$ & $\checkmark$ & - \\
$\Xi_{c c}^{+} \rightarrow \Omega_{c}^{(*) 0}+K^{(*)+}$ & $\ldots$ & $\ldots$ & $\checkmark$ & $\ldots$ & $\ldots$ \\
$\Xi_{c c}^{+} \rightarrow \Lambda^{0}\left(\Sigma^{(*) 0}\right)+D^{(*)+}$ & $\ldots$ & $\ldots$ & $\ldots$ & $\checkmark$ & $\checkmark$ \\
$\Xi_{c c}^{+} \rightarrow \Sigma^{(*)+}+D^{(*) 0}$ & $\ldots$ & $\ldots$ & - & $\ldots$ & $\checkmark$ \\
$\Xi_{c c}^{+} \rightarrow \Xi^{(*) 0}+D_{s}^{(*)+}$ & $\ldots$ & $\ldots$ & $\ldots$ & $\ldots$ & $\checkmark$ \\
$\Omega_{c c}^{+} \rightarrow \Xi_{c}^{(\prime, *)+}+\bar{K}^{(*) 0}$ & $\ldots$ & $\checkmark$ & $\ldots$ & $\checkmark$ & $\ldots$ \\
$\Omega_{c c}^{+} \rightarrow \Xi^{(*) 0}+D^{(*)+}$ & $\ldots$ & $\ldots$ & $\ldots$ & $\checkmark$ & $\ldots$ \\
$\Omega_{c c}^{+} \rightarrow \Omega_{c}^{(*) 0}+\pi^{+}\left(\rho^{+}\right)$ & $\checkmark$ & $\ldots$ & $\ldots$ & $\ldots$ & $\ldots$ \\
\hline \hline
\end{tabular}

$q_{i} q_{j}=q_{i}^{\prime} q_{j}^{\prime}$ is shared by the parent and daughter baryon $B_{1}$ and $B_{2}$, respectively. A sufficient condition for the factorizing class of decays is that (i) $q_{m}$ is not among $q_{1}, q_{2}, q_{3}$ and (ii) $q_{\bar{n}}$ is not among $q_{1}^{\prime}, q_{2}^{\prime}, q_{3}^{\prime}$. Using these two criteria we have identified the two groups of decays,

$$
\Xi_{c c}^{++} \rightarrow \Sigma_{c}^{(*)++}+\bar{K}^{(*) 0} \quad \Omega_{c c}^{+} \rightarrow \Omega_{c}^{(*) 0}+\pi^{+}\left(\rho^{+}\right),
$$

which proceed via the tree graphs alone.

(ii) The second class of decays involves in addition to the tree topologies the $W$-exchange topologies IIb which, however, do not contribute because of the Körner, Pati, and Woo (KPW) theorem $[39,40]$. The Körner, Pati, and Woo theorem states that the contraction of the flavor antisymmetric currentcurrent operator with a flavor symmetric final state configuration is 0 . There are two groups of decays that belong to this class given by

$$
\Xi_{c c}^{++} \rightarrow \Xi_{c}^{(*)+}+\pi^{+}\left(\rho^{+}\right) \quad \Omega_{c c}^{+} \rightarrow \Xi_{c}^{\prime(*)+}+\bar{K}^{(*) 0} .
$$

We neglect $\mathrm{SU}(3)$ breaking effects when applying the KPW theorem to the above two groups of decays. We plan to quantify the $\mathrm{SU}(3)$ breaking effects in a future dynamical calculation of these decays.

The recently observed decay $\Xi_{c c}^{++} \rightarrow \Xi_{c}^{+}+\pi^{+}$[2] is not discussed in this paper since, in addition to the tree diagram Ia, there is a nonvanishing contribution from the $W$ exchange diagram IIb. In this context it is interesting to observe that the decays

$\Xi_{c c}^{++} \rightarrow \Sigma^{*+}+D^{(*)+} \Xi_{c c}^{+} \rightarrow \Sigma^{* 0}+D^{(*)+} \Xi_{c c}^{+} \rightarrow \Sigma^{*+}+D^{(*) 0}$

$\Xi_{c c}^{+} \rightarrow \Xi^{* 0}+D_{s}^{(*)+} \quad \Omega_{c c}^{+} \rightarrow \Xi^{\prime(*) 0}+D^{(*)+}$ 
induced by the topologies IIb and III are predicted to be altogether 0 due to the KPW theorem. It would be very interesting to experimentally confirm this prediction.

Let us add a few comments concerning the $W$-exchange diagrams. The contribution of the $W$-exchange diagrams cannot be neglected even if this is frequently done in the analysis of nonleptonic charm baryon decays. A prominent example is the decay $\Lambda_{c}^{+} \rightarrow \Sigma^{0} \pi^{+}$which is contributed to by the topologies IIa, IIb, and III; i.e., there are no tree graph contributions to the decay. Nevertheless, its experimental branching ratio is comparable to that of the decay mode $\Lambda_{c}^{+} \rightarrow \Lambda^{0} \pi^{+}$where the latter mode is also contributed to by the tree diagram 1a. The interplay of the tree and $W$-exchange diagrams for the Cabibbo-favored $\Delta C=1$ nonleptonic charm baryon decays has been studied in [41-44] and also in a previous version of our model [45]. We hope to return to the calculation of the $W$-exchange contributions in single charm and double charm baryon decays in the framework of our CCQM quark model. We mention that the evaluation of the $W$-exchange diagrams in our approach is technically quite demanding since it involves a three-loop calculation. Naturally it is of utmost importance to get the relative signs between the tree and $W$-exchange contributions right since this decides whether the two classes of contributions interfere constructively or destructively. A first attempt to estimate the $W$-exchange contributions to the $1 / 2^{+} \rightarrow 1 / 2^{+}+0^{-}$double heavy baryon decays has been published in [25] using a baryon pole model for the $W$-exchange contributions. There is a one-to-one correspondence between the $1 / 2^{+} \rightarrow 1 / 2^{+}+0^{-}$decays treated in [25] and the $1 / 2^{+} \rightarrow 1 / 2^{+}+0^{-}$decays listed in Table I. The $W$-exchange topology structure of the decays written down in [25] in terms of $s$-channel and $u$-channel contributions is consistent with the corresponding topology structure in Table I.

Returning to the factorizing contributions we in the following discuss the class 1 and class 2 decays listed in Eqs. (2) and (3), respectively, which are determined by the factorizing contributions alone.

\section{MATRIX ELEMENTS, HELICITY AMPLITUDES AND DECAY RATES EXPRESSIONS}

The matrix element of the exclusive decay $B_{1}\left(p_{1}, \lambda_{1}\right) \rightarrow$ $B_{2}\left(p_{2}, \lambda_{2}\right)+M\left(q, \lambda_{M}\right)$ is defined by $\left(p_{1}=p_{2}+q\right)$

$$
\begin{aligned}
M\left(B_{1} \rightarrow B_{2}+M\right)= & \frac{G_{F}}{\sqrt{2}} V_{i j} V_{k l}^{*} C_{\text {eff }} f_{M} M_{M} \\
& \times\left\langle B_{2}\left|\bar{q}_{2} O_{\mu} q_{1}\right| B_{1}\right\rangle \epsilon^{\dagger \mu}\left(\lambda_{M}\right),
\end{aligned}
$$

where $M=V$ and $M=P$ stand for the vector and pseudoscalar meson cases such that $M_{M}$ and $f_{M}$ are the respective masses $M_{V}, M_{P}$ and leptonic decay constants $f_{V}, f_{P}$. The Dirac string $O^{\mu}$ is defined by $O^{\mu}=\gamma^{\mu}\left(1-\gamma^{5}\right)$. Here $V_{i j}$ are the Cabibbo-Kabayashi-Maskawa matrix elements: $V_{u d}=0.97420$ and $V_{c s}=0.997$.

Here $C_{\text {eff }}$ is the combination of the Wilson coefficients $\left(C_{2}+\xi C_{1}\right)$, where $\xi=1 / N_{c}$ and $N_{c}$ is the number of colors, while for neutral emission the color-flavor factor reads $\left(C_{1}+\xi C_{2}\right)$. We take $C_{1}=-0.51$ and $C_{2}=1.20$ at $\mu=m_{c}=1.3 \mathrm{GeV}$ from Ref. [38]. We use the large $N_{c}$ limit for the color-flavor factors. It is known that nonfactorizable contributions coming from, e.g., one-gluon exchange, might be important for the description of nonleptonic decays. As an example, recall the well-known decay $B \rightarrow J / \psi+K$, which is proportional to the coefficient $a_{2}=C_{1}+\xi C_{2}$ and would thus be predicted to be 0 for $N_{c}=3$. In this case naive factorization clearly does not describe the experimental data. The discussion on and determination of the nonfactorizable corrections to the coefficient $a_{2}$ has been actively pursued in the literature using various techniques. However, as far as we know, up to now there is no well-established framework in which the nonfactorizable contributions could be taken into account in a self-consistent way. Therefore, we employ a phenomenological and simple assumption in our calculations of the nonleptonic decays of both heavy mesons and baryons that the color factor $\xi=1 / N_{c}$ appearing in the combination of the Wilson coefficients is set to 0 . This assumption has been extensively used in the literature and is well justified in the comparison with experimental data in the meson sector.

The hadronic matrix element $\left\langle B_{2}\left|\bar{q}_{2} O^{\mu} q_{1}\right| B_{1}\right\rangle$ can be expressed in terms of six $\left(1 / 2^{+} \rightarrow 1 / 2^{+}\right)$and eight $\left(1 / 2^{+} \rightarrow 3 / 2^{+}\right)$dimensionless invariant form factors $F_{i}^{V / A}\left(q^{2}\right)$, respectively. One has for the transition $\frac{1}{2}^{+} \rightarrow \frac{1}{2}^{+}$,

$$
\begin{aligned}
\left\langle B_{2}\left|\bar{q}_{2} \gamma_{\mu} q_{1}\right| B_{1}\right\rangle & =\bar{u}\left(p_{2}, s_{2}\right)\left[\gamma_{\mu} F_{1}^{V}\left(q^{2}\right)-i \sigma_{\mu \nu} \frac{q_{\nu}}{M_{1}} F_{2}^{V}\left(q^{2}\right)+\frac{q_{\mu}}{M_{1}} F_{3}^{V}\left(q^{2}\right)\right] u\left(p_{1}, s_{1}\right), \\
\left\langle B_{2}\left|\bar{q}_{2} \gamma_{\mu} \gamma_{5} q_{1}\right| B_{1}\right\rangle & =\bar{u}\left(p_{2}, s_{2}\right)\left[\gamma_{\mu} F_{1}^{A}\left(q^{2}\right)-i \sigma_{\mu \nu} \frac{q_{\nu}}{M_{1}} F_{2}^{A}\left(q^{2}\right)+\frac{q_{\mu}}{M_{1}} F_{3}^{A}\left(q^{2}\right)\right] \gamma_{5} u\left(p_{1}, s_{1}\right)
\end{aligned}
$$

and for the transition $\frac{1}{2}^{+} \rightarrow \frac{3}{2}^{+}$, 


$$
\begin{aligned}
\left\langle B_{2}^{*}\left|\bar{q}_{2} \gamma_{\mu} q_{1}\right| B_{1}\right\rangle & =\bar{u}^{\alpha}\left(p_{2}, s_{2}\right)\left[g_{\alpha \mu} F_{1}^{V}\left(q^{2}\right)+\gamma_{\mu} \frac{p_{1 \alpha}}{M_{1}} F_{2}^{V}\left(q^{2}\right)+\frac{p_{1 \alpha} p_{2 \mu}}{M_{1}^{2}} F_{3}^{V}\left(q^{2}\right)+\frac{p_{1 \alpha} q_{\mu}}{M_{1}^{2}} F_{4}^{V}\left(q^{2}\right)\right] \gamma_{5} u\left(p_{1}, s_{1}\right), \\
\left\langle B_{2}^{*}\left|\bar{q}_{2} \gamma_{\mu} \gamma_{5} q_{1}\right| B_{1}\right\rangle & =\bar{u}^{\alpha}\left(p_{2}, s_{2}\right)\left[g_{\alpha \mu} F_{1}^{A}\left(q^{2}\right)+\gamma_{\mu} \frac{p_{1 \alpha}}{M_{1}} F_{2}^{A}\left(q^{2}\right)+\frac{p_{1 \alpha} p_{2 \mu}}{M_{1}^{2}} F_{3}^{A}\left(q^{2}\right)+\frac{p_{1 \alpha} q_{\mu}}{M_{1}^{2}} F_{4}^{A}\left(q^{2}\right)\right] u\left(p_{1}, s_{1}\right)
\end{aligned}
$$

where $\sigma_{\mu \nu}=(i / 2)\left(\gamma_{\mu} \gamma_{\nu}-\gamma_{\nu} \gamma_{\mu}\right)$ and all $\gamma$ matrices are defined as in Bjorken-Drell.

The results of a covariant dynamical calculation as in the present case are usually obtained in terms of the invariant form factors defined above. To proceed further, it is very convenient to convert the set of invariant form factors to a set of helicity amplitudes where the two sets are linearly related. We therefore express the vector and axial helicity amplitudes $H_{\lambda_{2} \lambda_{M}}^{V / A}$ in terms of the invariant form factors $F_{i}^{V / A}$, where $\lambda_{M}=t, \pm 1,0$ and $\lambda_{2}= \pm 1 / 2, \pm 3 / 2$ are the helicity components of the meson $M(M=P, V)$ and the baryon $B_{2}$, respectively. We need to calculate the expressions

$$
H_{\lambda_{2} \lambda_{M}}=\left\langle B_{2}\left(p_{2}, \lambda_{2}\right)\left|\bar{q}_{2} O_{\mu} q_{1}\right| B_{1}\left(p_{1}, \lambda_{1}\right)\right\rangle \epsilon^{\dagger \mu}\left(\lambda_{M}\right)=H_{\lambda_{2} \lambda_{M}}^{V}-H_{\lambda_{2} \lambda_{M}}^{A},
$$

where we split the helicity amplitudes into their vector and axial parts. For the color enhanced decays the operator $\bar{q}_{2} O_{\mu} q_{1}$ represents a charged current transition while, for the color suppressed decays, $\bar{q}_{2} O_{\mu} q_{1}$ describes a neutral current transition. We work in the rest frame of the baryon $B_{1}$ with the baryon $B_{2}$ moving in the positive $z$-direction: $p_{1}=\left(M_{1}, \overrightarrow{\mathbf{0}}\right), p_{2}=$ $\left(E_{2}, 0,0,\left|\mathbf{p}_{2}\right|\right)$ and $q=\left(q_{0}, 0,0,-\left|\mathbf{p}_{2}\right|\right)$. The helicities of the three particles are related by $\lambda_{1}=\lambda_{2}-\lambda_{M}$. We use the notation $\lambda_{P}=\lambda_{t}=0$ for the scalar $(J=0)$ contribution in order to set the helicity label apart from $\lambda_{V}=0$ used for the longitudinal component of the $J=1$ vector meson. The relations connecting the helicity amplitudes to the invariant form factors are given for the transition $\frac{1}{2}^{+} \rightarrow \frac{1}{2}^{+}: H_{-\lambda_{2},-\lambda_{M}}^{V}=+H_{\lambda_{2}, \lambda_{M}}^{V}$ and $H_{-\lambda_{2},-\lambda_{M}}^{A}=-H_{\lambda_{2}, \lambda_{M}}^{A}$.

$$
\begin{array}{ll}
H_{\frac{1}{2} t}^{V}=\sqrt{Q_{+} / q^{2}}\left(F_{1}^{V} M_{-}+F_{3}^{V} \frac{q^{2}}{M_{1}}\right) & H_{\frac{1}{2} t}^{A}=\sqrt{Q_{-} / q^{2}}\left(F_{1}^{A} M_{+}-F_{3}^{A} \frac{q^{2}}{M_{1}}\right) \\
H_{\frac{1}{2} 0}^{V}=\sqrt{Q_{-} / q^{2}}\left(F_{1}^{V} M_{+}+F_{2}^{V} \frac{q^{2}}{M_{1}}\right) & H_{\frac{1}{2} 0}^{A}=\sqrt{Q_{+} / q^{2}}\left(F_{1}^{A} M_{-}-F_{2}^{A} \frac{q^{2}}{M_{1}}\right) \\
H_{\frac{1}{2} 1}^{V}=\sqrt{2 Q_{-}}\left(-F_{1}^{V}-F_{2}^{V} \frac{M_{+}}{M_{1}}\right) & H_{\frac{1}{2} 1}^{A}=\sqrt{2 Q_{+}}\left(-F_{1}^{A}+F_{2}^{A} \frac{M_{-}}{M_{1}}\right),
\end{array}
$$

and for the transition $\frac{1}{2}^{+} \rightarrow \frac{3}{2}^{+}: H_{-\lambda_{2},-\lambda_{M}}^{V}=-H_{\lambda_{2}, \lambda_{M}}^{V}$ and $H_{-\lambda_{2},-\lambda_{M}}^{A}=+H_{\lambda_{2}, \lambda_{M}}^{A}$.

$$
\begin{aligned}
H_{\frac{1}{2} t}^{V} & =-\sqrt{\frac{2}{3} \cdot \frac{Q_{+}}{q^{2}}} \frac{Q_{-}}{2 M_{1} M_{2}}\left(F_{1}^{V} M_{1}-F_{2}^{V} M_{+}+F_{3}^{V} \frac{M_{+} M_{-}-q^{2}}{2 M_{1}}+F_{4}^{V} \frac{q^{2}}{M_{1}}\right) \\
H_{\frac{1}{2} 0}^{V} & =-\sqrt{\frac{2}{3} \cdot \frac{Q_{-}}{q^{2}}}\left(F_{1}^{V} \frac{M_{+} M_{-}-q^{2}}{2 M_{2}}-F_{2}^{V} \frac{Q_{+} M_{-}}{2 M_{1} M_{2}}+F_{3}^{V} \frac{\left|\mathbf{p}_{2}\right|^{2}}{M_{2}}\right) \\
H_{\frac{1}{2} 1}^{V} & =\sqrt{\frac{Q_{-}}{3}}\left(F_{1}^{V}-F_{2}^{V} \frac{Q_{+}}{M_{1} M_{2}}\right) \quad H_{\frac{3}{2} 1}^{V}=-\sqrt{Q_{-}} F_{1}^{V} \\
H_{\frac{1}{2} t}^{A} & =\sqrt{\frac{2}{3} \cdot \frac{Q_{-}}{q^{2}}} \frac{Q_{+}}{2 M_{1} M_{2}}\left(F_{1}^{A} M_{1}+F_{2}^{A} M_{-}+F_{3}^{A} \frac{M_{+} M_{-}-q^{2}}{2 M_{1}}+F_{4}^{A} \frac{q^{2}}{M_{1}}\right) \\
H_{\frac{1}{2} 0}^{A} & =\sqrt{\frac{2}{3} \cdot \frac{Q_{+}}{q^{2}}}\left(F_{1}^{A} \frac{M_{+} M_{-}-q^{2}}{2 M_{2}}+F_{2}^{A} \frac{Q_{-} M_{+}}{2 M_{1} M_{2}}+F_{3}^{A} \frac{\left|\mathbf{p}_{2}\right|^{2}}{M_{2}}\right) \\
H_{\frac{1}{2} 1}^{A} & =\sqrt{\frac{Q_{+}}{3}}\left(F_{1}^{A}-F_{2}^{A} \frac{Q_{-}}{M_{1} M_{2}}\right) \quad H_{\frac{3}{2} 1}^{A}=\sqrt{Q_{+}} F_{1}^{A} .
\end{aligned}
$$

We use the abbreviations $M_{ \pm}=M_{1} \pm M_{2}, Q_{ \pm}=M_{ \pm}^{2}-q^{2}$. The magnitude of the momentum of the daughter baryon $B_{2}$ is given by $\left|\mathbf{p}_{2}\right|=\sqrt{Q_{+} Q_{-}} / 2 M_{1}=\lambda^{1 / 2}\left(M_{1}^{2}, M_{2}^{2}, q^{2}\right) /\left(2 M_{1}\right)$. 
Let us add a few remarks on the helicity composition of the vector and axial vector helicity amplitudes. At the zero recoil point $q^{2}=\left(M_{1}-M_{2}\right)^{2}$ the vector helicity amplitudes vanish and the transverse-to-longitudinal composition can be seen to be given by $\mathcal{F}_{L} / \mathcal{F}_{T}=1 / 2$ for both $1 / 2^{+} \rightarrow 1 / 2^{+}, 3 / 2^{+}$transitions ("allowed Fermi-Teller transition"). At the other end of the $q^{2}$-spectrum at $q^{2}=0$ the longitudinal mode dominates. These findings have a bearing on the transverse-to-longitudinal composition of the vector mesons in the nonleptonic decays to be discussed later on.

Using the helicity amplitudes one can write down very compact expressions for the various decay rates. The semileptonic decay width is given by $\left(m_{\ell}=0\right)$

$$
\begin{aligned}
& \Gamma\left(B_{1} \rightarrow B_{2}+\ell^{+} \nu_{\ell}\right) \\
& \quad=\int_{0}^{\left(M_{1}-M_{2}\right)^{2}} d q^{2} \frac{d \Gamma\left(B_{1} \rightarrow B_{2}+\ell^{+} \nu_{\ell}\right)}{d q^{2}}
\end{aligned}
$$

where

$$
\frac{d \Gamma\left(B_{1} \rightarrow B_{2}+\ell^{+} \nu_{\ell}\right)}{d q^{2}}=\frac{1}{192 \pi} G_{F}^{2} \frac{\left|\mathbf{p}_{2}\right| q^{2}}{M_{1}^{2}}\left|V_{i j}\right|^{2} \mathcal{H}_{V}\left(\mathcal{H}_{V}^{\prime}\right)
$$

For the nonleptonic decays one has

$$
\Gamma\left(B_{1} \rightarrow B_{2}+V\right)=\frac{G_{F}^{2}}{32 \pi} \frac{\left|\mathbf{p}_{2}\right|}{M_{1}^{2}}\left|V_{i j} V_{k l}^{*}\right|^{2} C_{\text {eff }}^{2} f_{V}^{2} M_{V}^{2} \mathcal{H}_{V}\left(\mathcal{H}_{V}^{\prime}\right),
$$

$\Gamma\left(B_{1} \rightarrow B_{2}+P\right)=\frac{G_{F}^{2}}{32 \pi} \frac{\left|\mathbf{p}_{2}\right|}{M_{1}^{2}}\left|V_{i j} V_{k l}^{*}\right|^{2} C_{\mathrm{eff}}^{2} f_{P}^{2} M_{P}^{2} \mathcal{H}_{S}\left(\mathcal{H}_{S}^{\prime}\right)$,

where we denote the sum of the squared moduli of the helicity amplitudes by $\mathcal{H}_{V}, \mathcal{H}_{S}, \mathcal{H}_{V}^{\prime}$ and $\mathcal{H}_{S}^{\prime}$ according to the two cases

$$
\begin{aligned}
1 / 2^{+} \rightarrow 1 / 2^{+}: \mathcal{H}_{V} & =\sum_{\lambda_{2}= \pm 1 / 2, \lambda_{V}= \pm 1,0}\left|H_{\lambda_{2}, \lambda_{V}}\right|^{2} \\
\mathcal{H}_{S} & =\sum_{\lambda_{2}= \pm 1 / 2}\left|H_{\lambda_{2}, \lambda_{t}}\right|^{2} \\
1 / 2^{+} \rightarrow 3 / 2^{+}: \mathcal{H}_{V}^{\prime} & =\sum_{\lambda_{2}= \pm 1 / 2, \pm 3 / 2, \lambda_{V}= \pm 1,0}\left|H_{\lambda_{2}, \lambda_{V}}\right|^{2} \\
\mathcal{H}_{S}^{\prime} & =\sum_{\lambda_{2}= \pm 1 / 2}\left|H_{\lambda_{2}, \lambda_{t}}\right|^{2} .
\end{aligned}
$$

Angular momentum conservation dictates the constraint $\left|\lambda_{2}-\lambda_{M}\right| \leq 1 / 2$ for the helicity amplitudes since the initial state baryon has spin $1 / 2$.
It is quite convenient to work with normalized helicity amplitudes which we denote by $\hat{H}_{\lambda_{2} \lambda_{M}}$. The helicity amplitudes are normalized according to

$$
\hat{H}_{\lambda_{2} t}=H_{\lambda_{2} t} / \mathcal{H}_{S}^{1 / 2} \quad \hat{H}_{\lambda_{2} \lambda_{V}}=H_{\lambda_{2} \lambda_{V}} / \mathcal{H}_{V}^{1 / 2}
$$

for the $1 / 2^{+} \rightarrow 1 / 2^{+}$case and accordingly for the $1 / 2^{+} \rightarrow$ $3 / 2^{+}$case with $\mathcal{H}_{S} \rightarrow \mathcal{H}_{S}^{\prime}$ and $\mathcal{H}_{V} \rightarrow \mathcal{H}_{V}^{\prime}$.

\section{INTERPOLATING CURRENTS AND CALCULATION OF THE TRANSITION FORM FACTORS IN THE CCQM}

As described in the introduction we use the CCQM to calculate the various $1 / 2^{+} \rightarrow 1 / 2^{+}$and $1 / 2^{+} \rightarrow 3 / 2^{+}$ transition form factors $F_{i}^{V, A}\left(q^{2}\right)$ that are needed in the calculation of the helicity amplitudes. We describe the coupling of the baryons with the constituent quarks by nonlocal extensions of the interpolating currents (see details in Refs. [7,10,45-50]). In Table II we list the interpolating currents needed in the present application.

The three constituent quarks are treated as separate dynamic entities which propagate with fully covariant fermion propagators $S_{q}(k)=1 /\left(m_{q}-\not k\right)$ in the two-loop Feynman diagram which describes the current-induced transition between the respective baryons. The propagator masses $m_{q}$ are constituent quark masses fixed in previous analyses of a multitude of hadronic processes within our approach (see, e.g., Refs. [46,47]).

Apart from the choice of the interpolating current and the constituent quark masses there are two parameters that describe the structure of a baryon in the CCQM. These are the coupling factor of the baryon to its constituent quarks $g_{B}$ and the size parameters $\Lambda_{B}$ characterizing the size of the

TABLE II. Cabibbo-favored nonleptonic two-body decays of double heavy charm baryons including $W$-exchange contributions.

\begin{tabular}{llll}
\hline \hline Baryon & $J^{P}$ & Interpolating current & Mass [MeV] \\
\hline$\Xi_{c c}^{++}$ & $\frac{1}{2}^{+}$ & $\epsilon^{a b c} \gamma^{\mu} \gamma_{5} u^{a} c^{b} C \gamma_{\mu} c^{c}$ & 3620.6 \\
$\Xi_{c c}^{+}$ & $\frac{1}{2}{ }^{+}$ & $\epsilon^{a b c} \gamma^{\mu} \gamma_{5} d^{a} c^{b} C \gamma_{\mu} c^{c}$ & 3620.6 \\
$\Omega_{c c}^{+}$ & $\frac{1}{2}^{+}$ & $\epsilon^{a b c} \gamma^{\mu} \gamma_{5} s^{a} c^{b} C \gamma_{\mu} c^{c}$ & 3710 \\
$\Sigma_{c}^{++}$ & $\frac{1}{2}{ }^{+}$ & $\epsilon^{a b c} \gamma^{\mu} \gamma_{5} c^{a} u^{b} C \gamma_{\mu} u^{c}$ & 2453.97 \\
$\Sigma_{c}^{*++}$ & $\frac{3}{2}^{+}$ & $\epsilon^{a b c} c^{a} u^{b} C \gamma_{\mu} u^{c}$ & 2518.41 \\
$\Xi_{c}^{+}$ & $\frac{1}{2}{ }^{+}$ & $\epsilon^{a b c} c^{a} u^{b} C \gamma_{5} s^{c}$ & 2467.93 \\
$\Xi_{c}^{\prime+}$ & $\frac{1}{2}{ }^{+}$ & $\epsilon^{a b c} \gamma^{\mu} \gamma_{5} c^{a} u^{b} C \gamma_{\mu} s^{c}$ & 2577.4 \\
$\Xi_{c}^{0}$ & $\frac{1}{2}{ }^{+}$ & $\epsilon^{a b c} c^{a} d^{b} C \gamma_{5} s^{c}$ & 2470.85 \\
$\Xi_{c}^{\prime 0}$ & $\frac{1}{2}{ }^{+}$ & $\epsilon^{a b c} \gamma^{\mu} \gamma_{5} c^{a} d^{b} C \gamma_{\mu} s^{c}$ & 2577.9 \\
$\Xi_{c}^{*+}$ & $\frac{3}{2}{ }^{+}$ & $\epsilon^{a b c} c^{a} u^{b} C \gamma_{\mu} s^{c}$ & 2645.57 \\
$\Xi_{c}^{* 0}$ & $\frac{3}{2}{ }^{+}$ & $\epsilon^{a b c} c^{a} d^{b} C \gamma_{\mu} s^{c}$ & 2646.38 \\
$\Omega_{c}^{0}$ & $\frac{1}{2}{ }^{+}$ & $\epsilon^{a b c} \gamma^{\mu} \gamma_{5} c^{a} s^{b} C \gamma_{\mu} s^{c}$ & 2695.2 \\
$\Omega_{c}^{* 0}$ & $\frac{3}{2}{ }^{+}$ & $\epsilon^{a b c} c^{a} s^{b} C \gamma_{\mu} s^{c}$ & 2765.9 \\
\hline \hline
\end{tabular}


nonlocal distribution of the quarks in the baryons. The coupling factor $g_{B}$ and the size parameter $\Lambda_{B}$ become related by the compositeness condition of Salam and Weinberg [51,52]. By analogy we treat mesons as bound states of a constituent quark and an antiquark; i.e., we construct respective nonlocal interaction Lagrangians of mesons with their constituent quarks (see details in Refs. [35,49]).

The details of calculating the $1 / 2^{+} \rightarrow 1 / 2^{+}$and $1 / 2^{+} \rightarrow$ $3 / 2^{+}$transition form factors between baryons have been discussed in detail in Refs. [7,10,45-50], and need not be repeated here.

\section{POLARIZATION, LONGITUDINAL/ TRANSVERSE HELICITY FRACTIONS AND ANGULAR DECAY DISTRIBUTIONS}

Since the semileptonic and nonleptonic two-body decays of the $\Xi_{c c}^{++}, \Xi_{c c}^{+}$and $\Omega_{c c}^{+}$are mediated by weak interactions, one can expect sizable polarization effects in these decays entailing nontrivial angular decay distributions in the decays of the mesons and baryons further down the decay chains. We treat the initial state baryons $\Xi_{c c}^{++}, \Xi_{c c}^{+}$and $\Omega_{c c}^{+}$ as being unpolarized. In principle, the parent baryons could acquire a nonzero transverse polarization in the hadronic production process which would depend on the rapidity of the baryon in question. However, since one is usually averaging over the rapidities of the production process, the parent baryons become effectively unpolarized (for more details see [47]).

\section{A. Semileptonic decays}

We only consider the Cabibbo-favored semileptonic decays of the double heavy charm baryons $\Xi_{c c}^{++}, \Xi_{c c}^{+}$ and $\Omega_{c c}^{+}$induced by the quark level $c \rightarrow s$ transition. The Cabibbo-suppressed semileptonic decays induced by the quark level $c \rightarrow d$ transitions are suppressed by an overall factor $\left(V_{c d} / V_{c s}\right)^{2}=0.049$. The Cabibbo suppression factor $\left(V_{c d} / V_{c s}\right)^{2}$ is partly offset by the larger phase space of the $\Delta S=1$ Cabibbo-suppressed decays which then amounts to an overall suppression factor of $\sim 0.1$ (see e.g., [31]).

The $\mathrm{Q}$ values of the semileptonic $c \rightarrow s$ decays discussed here are not large enough to allow for the semileptonic $\tau$ modes. On the other hand, the $\mathrm{Q}$ values are sufficiently large to allow one to neglect the lepton masses in the semileptonic $e^{+}$- and $\mu^{+}$-modes.

In Table III we present our numerical results for the Cabibbo-favored semileptonic decays of the double heavy charm baryon states $\Xi_{c c}^{++}, \Xi_{c c}^{+}$and $\Omega_{c c}^{+}$. We also list branching fractions for the semileptonic decays of the $\Xi_{c c}^{++}$based on the recent measurement of the lifetime of the $\Xi_{c c}^{++}[3]: \tau_{\Xi_{c c}^{++}}=\left(256_{-22}^{+24} \pm 14\right)$ fs.

For the semileptonic decays of the $\Xi_{c c}^{+}$and $\Omega_{c c}^{+}$we quote nominal branching fractions. These are nominal since the lifetimes of the double heavy charm baryon states $\Xi_{c c}^{+}$ and $\Omega_{c c}^{+}$have not been measured yet. One has to rely on theoretical calculations $[14,19]$ from which we take the median values

$$
\tau_{\Xi_{c c}^{+}}=190 \mathrm{fs}, \quad \tau_{\Omega_{c c}^{+}}=210 \mathrm{fs} .
$$

In the case that the experimental lifetime of the $\Xi_{c c}^{++}$ changes in the future and the lifetimes of the $\Xi_{c c}^{+}$and $\Omega_{c c}^{+}$become known one has to rescale our branching fractions by the ratios

$$
\left(\frac{\tau_{\Xi_{c c}^{++}}}{256 \mathrm{fs}}\right), \quad\left(\frac{\tau_{\Xi_{c c}^{+}}}{190 \mathrm{fs}}\right), \quad\left(\frac{\tau_{\Xi_{c c}^{++}}}{210 \mathrm{fs}}\right) .
$$

In Table III we also include numerical values for the $q^{2}$-averages of the transverse-plus, longitudinal and transverse-minus helicity fractions of the off-shell gauge boson $W^{-}$where we denote the averages by $\left\langle\mathcal{F}_{+}\right\rangle,\left\langle\mathcal{F}_{0}\right\rangle$ and $\left\langle\mathcal{F}_{-}\right\rangle$. When taking the $q^{2}$-averages one has to integrate the numerators and denominators separately including the factor $\left|\mathbf{p}_{2}\right| q^{2}$ [see Eq. (12)]. The $q^{2}$-dependent helicity fractions are defined by

TABLE III. Cabibbo-favored semileptonic decays of double heavy charm baryons induced by the charm level $c \rightarrow s$ transition $\left(\ell=e^{+}, \mu^{+}\right)$.

\begin{tabular}{llccccc}
\hline \hline & & $\Gamma\left[10^{-13} \mathrm{GeV}\right]$ & $\mathcal{B}[\%]$ & $\left\langle\mathcal{F}_{+}\right\rangle$ & $\left\langle\mathcal{F}_{0}\right\rangle$ & $\left\langle\mathcal{F}_{-}\right\rangle$ \\
\hline $1 / 2^{+} \rightarrow 1 / 2^{+}$ & $\Xi_{c c}^{++} \rightarrow \Xi_{c}^{+}+\ell^{+} \nu_{\ell}$ & 0.70 & 2.72 & 0.02 & 0.88 & 0.10 \\
& $\Xi_{c c}^{++} \rightarrow \Xi_{c}^{\prime+}+\ell^{+} \nu_{\ell}$ & 0.97 & 3.76 & 0.09 & 0.55 & 0.36 \\
& $\Xi_{c c}^{+} \rightarrow \Xi_{c}^{0}+\ell^{+} \nu_{\ell}$ & 0.69 & 2.00 & 0.02 & 0.88 & 0.10 \\
& $\Xi_{c c}^{+} \rightarrow \Xi_{c}^{\prime 0}+\ell^{+} \nu_{\ell}$ & 0.97 & 2.79 & 0.09 & 0.55 & 0.36 \\
& $\Omega_{c c}^{+} \rightarrow \Omega_{c}^{0}+\ell^{+} \nu_{\ell}$ & 1.82 & 7.07 & 0.09 & 0.55 & 0.36 \\
$1 / 2^{+} \rightarrow 3 / 2^{+}$ & $\Xi_{c c}^{++} \rightarrow \Xi_{c}^{*+}+\ell^{+} \nu_{\ell}$ & 0.22 & 0.86 & 0.12 & 0.49 & 0.39 \\
& $\Xi_{c c}^{+} \rightarrow \Xi_{c}^{* 0}+\ell^{+} \nu_{\ell}$ & 0.22 & 0.64 & 0.12 & 0.49 & 0.39 \\
& $\Omega_{c c}^{+} \rightarrow \Omega_{c}^{* 0}+\ell^{+} \nu_{\ell}$ & 0.40 & 1.27 & 0.12 & 0.49 & 0.39 \\
\hline \hline
\end{tabular}




$$
\begin{aligned}
& 1 / 2^{+} \rightarrow 1 / 2^{+}: \mathcal{F}_{+}\left(q^{2}\right)=\left|\hat{H}_{1 / 2,1}\right|^{2} \quad \mathcal{F}_{0}\left(q^{2}\right)=\left|\hat{H}_{1 / 2,0}\right|^{2}+\left|\hat{H}_{-1 / 2,0}\right|^{2} \quad \mathcal{F}_{-}\left(q^{2}\right)=\left|\hat{H}_{-1 / 2,-1}\right|^{2} \\
& 1 / 2^{+} \rightarrow 3 / 2^{+}: \mathcal{F}_{+}\left(q^{2}\right)=\sum_{\lambda_{2}}\left|\hat{H}_{\lambda_{2}, 1}\right|^{2} \quad \mathcal{F}_{0}\left(q^{2}\right)=\left|\hat{H}_{1 / 2,0}\right|^{2}+\left|\hat{H}_{-1 / 2,0}\right|^{2} \quad \mathcal{F}_{-}\left(q^{2}\right)=\sum_{\lambda_{2}}\left|\hat{H}_{\lambda_{2},-1}\right|^{2},
\end{aligned}
$$

where $\lambda_{2}=1 / 2,3 / 2$ for $\mathcal{F}_{+}\left(q^{2}\right)$ and $\lambda_{2}=-1 / 2,-3 / 2$ for $\mathcal{F}_{-}\left(q^{2}\right)$ in the $1 / 2^{+} \rightarrow 3 / 2^{+}$case. Since we use normalized helicity amplitudes $\hat{H}_{\lambda_{2} \lambda_{M}}$ [see Eq. (16)] the helicity fractions satisfy $\mathcal{F}_{+}\left(q^{2}\right)+\mathcal{F}_{0}\left(q^{2}\right)+\mathcal{F}_{-}\left(q^{2}\right)=1$. The angular decay distribution of the lepton $\ell^{-}$in the $\left(\ell^{-} ; \bar{\nu}_{\ell}\right)$ rest frame is given by

$$
\begin{aligned}
W(\theta)= & \frac{3}{8}(1-\cos \theta)^{2} \mathcal{F}_{+}+\frac{3}{4} \sin ^{2} \theta \mathcal{F}_{0} \\
& +\frac{3}{8}(1+\cos \theta)^{2} \mathcal{F}_{-},
\end{aligned}
$$

where the angle $\theta$ is defined in analogy to the angle $\theta_{V}$ in Fig. 2 with the change of labeling $\left(K^{-} \rightarrow \ell^{-}\right),\left(\pi^{+} \rightarrow \bar{\nu}_{\ell}\right)$ and $\left(\bar{K}^{* 0} \rightarrow W_{\text {off-shell }}^{-}\right)$. We do not discuss polarization effects on the hadron side of the semileptonic decays. These can be discussed along the lines of [48].

\section{B. Nonleptonic decays}

We discuss the rates, branching fractions and angular decay distributions of the four classes of decays,

$$
\begin{aligned}
& 1 / 2^{+} \rightarrow 1 / 2^{+}+0^{-} \\
& 1 / 2^{+} \rightarrow 1 / 2^{+}+1^{-} \\
& 1 / 2^{+} \rightarrow 3 / 2^{+}+0^{-} \\
& 1 / 2^{+} \rightarrow 3 / 2^{+}+1^{-} .
\end{aligned}
$$

Each of the above classes contains four factorizing nonleptonic two-body decays. Thus we discuss altogether 16 factorizing nonleptonic two-body decays comprising the decays $\Xi_{c c}^{++} \rightarrow \Sigma_{c}^{(*)++}+\bar{K}^{(*) 0}, \quad \Omega_{c c}^{+} \rightarrow \Omega_{c}^{(*) 0}+\pi^{+}\left(\rho^{+}\right)$,

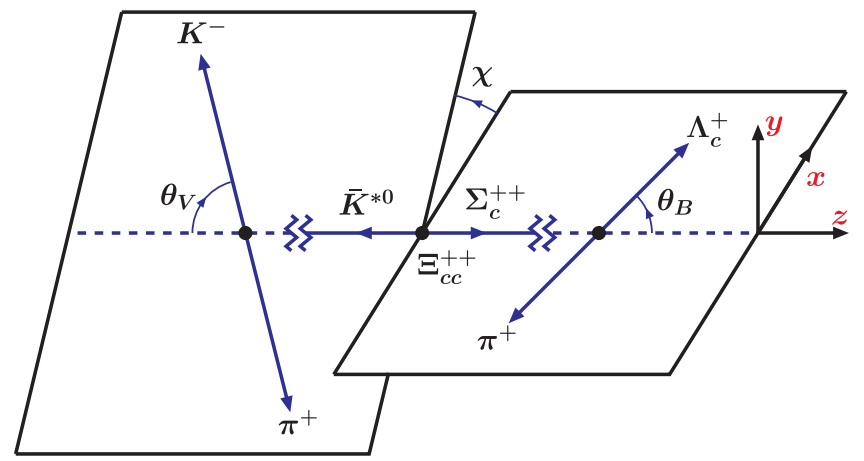

FIG. 2. Definition of the angles $\theta_{B}, \theta_{V}$, and $\chi$ in the cascade decay $\Xi_{c c}^{++} \rightarrow \Sigma_{c}^{++}\left(\rightarrow \Lambda_{c}^{+} \pi^{+}\right)+\bar{K}^{0 *}\left(\rightarrow K^{-} \pi^{+}\right)$. The $\Sigma_{c}^{++}$can either be the $\Sigma_{c}^{++}\left(2455 ; 1 / 2^{+}\right)$or the $\Sigma_{c}^{++}\left(2520 ; 3 / 2^{+}\right)$.
$\Xi_{c c}^{++} \rightarrow \Xi_{c}^{\prime(*)+}+\pi^{+}\left(\rho^{+}\right)$and $\Omega_{c c}^{+} \rightarrow \Xi_{c}^{\prime(*)+}+\bar{K}^{(*) 0}$ as they appear in rows 1, 2, 13 and 15 of Table I.

When discussing angular decay distributions we concentrate on the $1 / 2^{+} \rightarrow 1 / 2^{+}+1^{-}$two-sided cascade decay $\Xi_{c c}^{++} \rightarrow \Sigma_{c}^{++}\left(2455 ; 1 / 2^{+}\right)\left(\rightarrow \Lambda_{c}^{+} \pi^{+}\right)+\bar{K}^{* 0}\left(\rightarrow K^{-} \pi^{+}\right)$ and the $1 / 2^{+} \rightarrow 3 / 2^{+}+1^{-}$cascade decay $\Xi_{c c}^{++} \rightarrow$ $\Sigma_{c}^{++}\left(2520 ; 3 / 2^{+}\right)\left(\rightarrow \Lambda_{c}^{+} \pi^{+}\right)+\bar{K}^{* 0}\left(\rightarrow K^{-} \pi^{+}\right)$as well as the corresponding two one-sided cascade decays with $\bar{K}^{* 0}$ replaced by $\bar{K}^{0}$. These decay chains are favored from an experimental point of view since the second stage branching ratios are large. On the baryon side the daughter baryon decays $\Sigma_{c}^{++}\left(2455,1 / 2^{+}\right) \rightarrow \Lambda_{c}^{+} \pi^{+}$and $\Sigma_{c}^{++}(2520$; $\left.3 / 2^{+}\right) \rightarrow \Lambda_{c}^{+} \pi^{+}$have a large branching ratio close to $100 \%$. On the meson side the branching ratio of the decay $\bar{K}^{* 0} \rightarrow$ $K^{-} \pi^{+}$is also quite large ( $\sim 66 \%$ from isospin invariance). Further, all final states in the decay chains are charged, which is optimal from an experimental point of view.

In the following we discuss the classes of decays separately:

(i) $1 / 2^{+} \rightarrow 1 / 2^{+}+0^{-}$

In Table IV we list the rates, branching fractions and polarization of the daughter baryon $P_{B_{2}}$ for the four decays in this class.

As mentioned before we concentrate on the cascade decay $\Xi_{c c}^{++} \rightarrow \Sigma_{c}^{++}\left(\rightarrow \Lambda_{c}^{+} \pi^{+}\right)+\bar{K}^{0}$ when discussing polarization effects and angular decay distributions. The stage 2 decay $\Sigma_{c}^{++} \rightarrow \Lambda_{c}^{+} \pi^{+}$is a parity-conserving strong decay such that the onefold angular decay distribution of this cascade decay is given by

$$
\begin{aligned}
W\left(\theta_{B}\right) & =\sum_{\lambda_{2}, \lambda_{3}= \pm 1 / 2} \hat{H}_{\lambda_{2} t} \hat{H}_{\lambda_{2} t}^{*} d_{\lambda_{2} \lambda_{3}}^{1 / 2}\left(\theta_{B}\right) d_{\lambda_{2} \lambda_{3}}^{1 / 2}\left(\theta_{B}\right) \\
& =\sum_{\lambda_{2}= \pm 1 / 2} \hat{H}_{\lambda_{2} t} \hat{H}_{\lambda_{2} t}^{*}=1,
\end{aligned}
$$

where the polar angle $\theta_{B}$ is defined in Fig. 2.

When simplifying the decay distribution (22) we have used the orthonormality relation for the spin $1 / 2$ Wigner $d^{1 / 2}$-function

$$
\sum_{\lambda_{3}} d_{\lambda_{2} \lambda_{3}}^{1 / 2}\left(\theta_{B}\right) d_{\lambda_{2}^{\prime} \lambda_{3}}^{1 / 2}\left(\theta_{B}\right)=\delta_{\lambda_{2} \lambda_{2}^{\prime}}
$$

The bilinear forms of the helicity amplitudes sum up to 1 since normalized helicity amplitudes are used. The angular decay distribution (22) can be seen to be flat. 
TABLE IV. Cabibbo-favored factorizing nonleptonic two-body decays of double heavy charm baryons induced by the quark level $c \rightarrow s ; d \rightarrow u$ transitions for the cases $1 / 2^{+} \rightarrow 1 / 2^{+}+0^{-}$.

\begin{tabular}{llccc}
\hline \hline & & $\Gamma\left[10^{-13} \mathrm{GeV}\right]$ & $\mathcal{B}[\%]$ & $P_{B_{2}}$ \\
\hline $1 / 2^{+} \rightarrow 1 / 2^{+}+0^{-}$ & $\Xi_{c c}^{++} \rightarrow \Sigma_{c}^{++}+\bar{K}^{0}$ & 0.32 & 1.25 & -0.96 \\
& $\Xi_{c c}^{++} \rightarrow \Xi_{c}^{\prime+}+\pi^{+}$ & 0.78 & 3.03 & -0.94 \\
& $\Omega_{c c}^{+} \rightarrow \Xi_{c}^{\prime+}+\bar{K}^{0}$ & 0.17 & 0.54 & -0.97 \\
& $\Omega_{c c}^{+} \rightarrow \Omega_{c}^{0}++\pi^{+}$ & 1.58 & 5.05 & -0.94 \\
\hline \hline
\end{tabular}

The longitudinal polarization of the daughter baryon $\Sigma_{c}^{++}$in the stage 1 decay $\Xi_{c c}^{++} \rightarrow \Sigma_{c}^{++}+$ $\bar{K}^{0}$ is given by

$$
P_{\Sigma_{c}^{++}}=\left|\hat{H}_{\frac{1}{2} t}\right|^{2}-\left|\hat{H}_{-\frac{1}{2}}\right|^{2} .
$$

We refer to this polarization parameter as $P_{B_{2}}$ in the general context and list $P_{B_{2}}$ for the other three decays in Table IV. As discussed after (22) the polarization of the daughter baryon $P_{\Sigma_{c}^{++}}$cannot be resolved from its angular decay distribution because the decay $\Sigma_{c}^{++} \rightarrow \Lambda_{c}^{+} \pi^{+}$is a strong decay. However, the polarization of the $\Sigma_{c}^{++}$is transferred to the second stage baryon $\Lambda_{c}^{+}$. The degree of polarization transfer depends on the baryon side polar angle $\theta_{B}$ and is given by

$$
P_{\Lambda_{c}^{+}}=P_{\Sigma_{c}^{++}} \cos \theta_{B}
$$

The polarization of the $\Lambda_{c}^{+}$can in turn be analyzed through its weak decays as e.g., in the decay $\Lambda_{c}^{+} \rightarrow \Lambda^{0} \pi^{+}$, which possesses a large analyzing power of $-0.91 \pm 0.15$ [36]. From an experimental point of view the decay $\Lambda_{c}^{+} \rightarrow p K^{-} \pi^{+}$would be preferred as an analyzing channel since it has a larger branching fraction than the decay $\Lambda_{c}^{+} \rightarrow$ $\Lambda^{0} \pi^{+}$by a factor of $\sim 5$. However, to our knowledge the analyzing power of this mode has neither been measured experimentally nor calculated theoretically except for an analysis of the two subchannels $\Lambda_{c}^{+} \rightarrow p \bar{K}^{* 0}$ and $\Lambda_{c}^{+} \rightarrow \Delta^{++} K^{-}$[53].

The decays $\Xi_{c c}^{++} \rightarrow \Xi_{c}^{\prime+}+\pi^{+}$and $\Omega_{c c}^{+} \rightarrow \Xi_{c}^{\prime+}+$ $\bar{K}^{0}$ involve daughter charm baryon state $\Xi_{c}^{\prime+}$, which then cascades down to the ground state $\Xi_{c}^{+}$ via a parity-conserving one-photon emission $\Xi_{c}^{\prime+} \rightarrow$ $\Xi_{c}^{+}+\gamma$. As in the decay $\Xi_{c c}^{++} \rightarrow \Sigma_{c}^{++}\left(\rightarrow \Lambda_{c}^{+} \pi^{+}\right)+$ $\bar{K}^{0}$ discussed above the helicity angle distribution of the $\Xi_{c}^{+}$is flat. Differing from Eq. (25) the polarization transfer is now

$$
P_{\Xi_{c}^{+}}=-P_{\Xi_{c}^{\prime+}} \cos \theta_{B}
$$

As concerns the decay $\Omega_{c c}^{+} \rightarrow \Omega_{c}^{0}+\pi^{+}$the daughter baryon $\Omega_{c}^{0}$ has a multitude of decay channels of which the relevant decay asymmetries have not been determined yet experimentally. Theoretical predictions for the two-body decay asymmetries of the daughter baryon $\Omega_{c}^{0}$ can be found in Refs. [41,42].

(ii) $1 / 2^{+} \rightarrow 1 / 2^{+}+1^{-}$

In Table $\mathrm{V}$ we list the rates, branching fractions, polarization of the daughter baryon $P_{B_{2}}$ and helicity fractions of the vector meson for the four decays in this class.

The threefold angular decay distribution for the generic cascade decay $1 / 2^{+} \rightarrow 1 / 2^{+}\left(\rightarrow 1 / 2^{+}+0^{-}\right)+$ $1^{-}\left(\rightarrow 0^{-}+0^{-}\right)$is given by

$$
\begin{aligned}
& W\left(\theta_{B}, \theta_{V}, \chi\right) \\
& =\sum_{\lambda_{V}, \lambda_{V}^{\prime} \lambda_{2}, \lambda_{2}^{\prime}, \lambda_{3}} \delta_{\lambda_{2}-\lambda_{V}, \lambda_{2}^{\prime}-\lambda_{V}^{\prime}} e^{-i\left(\lambda_{V}-\lambda_{V}^{\prime}\right) \chi} d_{0, \lambda_{V}}^{1}\left(\theta_{V}\right) d_{0 \lambda_{V}^{\prime}}^{1}\left(\theta_{V}\right) \\
& \quad \times \hat{H}_{\lambda_{2} \lambda_{V}} \hat{H}_{\lambda_{2}^{\prime} \lambda_{V}^{\prime}}^{*} d_{\lambda_{2} \lambda_{3}}^{1 / 2}\left(\theta_{B}\right) d_{\lambda_{2} \lambda_{3}}^{1 / 2}\left(\theta_{B}\right) \\
& =\sum_{\lambda_{V}, \lambda_{2}} d_{0, \lambda_{V}}^{1}\left(\theta_{V}\right) d_{0 \lambda_{V}}^{1}\left(\theta_{V}\right) \hat{H}_{\lambda_{2} \lambda_{V}} \hat{H}_{\lambda_{2} \lambda_{V}}^{*}
\end{aligned}
$$

where we have assumed that the stage 2 decays on the baryon and meson side are strong and thus parity conserving as in the cascade decay

TABLE V. Cabibbo-favored factorizing nonleptonic two-body decays of double heavy charm baryons induced by the quark level $c \rightarrow s ; d \rightarrow u$ transitions for the case $1 / 2^{+} \rightarrow 1 / 2^{+}+1^{-}$.

\begin{tabular}{llccccc}
\hline \hline & & $\Gamma\left[10^{-13} \mathrm{GeV}\right]$ & $\mathcal{B}[\%]$ & $\mathcal{F}_{L}$ & $\mathcal{F}_{T}$ & $P_{B_{2}}$ \\
\hline $1 / 2^{+} \rightarrow 1 / 2^{+}+1^{-}$ & $\Xi_{c c}^{++} \rightarrow \Sigma_{c}^{++}+\bar{K}^{* 0}$ & 1.44 & 5.61 & 0.47 & 0.53 & -0.82 \\
& $\Xi_{c c}^{++} \rightarrow \Xi_{c}^{\prime+}+\rho^{+}$ & 4.14 & 16.10 & 0.49 & 0.51 & -0.74 \\
& $\Omega_{c c}^{+} \rightarrow \Xi_{c}^{\prime+}+\bar{K}^{* 0}$ & 0.75 & 2.39 & 0.45 & 0.55 & -0.79 \\
& $\Omega_{c c}^{+} \rightarrow \Omega_{c}^{0}+\rho^{+}$ & 8.29 & 26.44 & 0.48 & 0.52 & -0.71 \\
\hline \hline
\end{tabular}


$\Sigma_{c}^{++}\left(2455 ; 1 / 2^{+}\right)\left(\rightarrow \Lambda_{c}^{+} \pi^{+}\right)+\bar{K}^{* 0}\left(\rightarrow K^{-} \pi^{+}\right)$which we focus on in the following. When evaluating the helicity sum in (27) one has to keep in mind that $\left|\lambda_{2}-\lambda_{V}\right| \leq 1 / 2$. The three angles $\theta_{V}, \theta_{B}$ and $\chi$ describing the angular structure of the decay are defined in Fig. 2. We have again used the orthonormality property Eq. (23) in the reduction of the first row of Eq. (27). The seeming threefold angular decay distribution has collapsed to a onefold angular decay distribution. In particular, the azimuthal correlation between the two decay planes spanned by $\left\{\Lambda_{c}^{+}, \pi^{+}\right\}$and $\left\{K^{-}, \pi^{+}\right\}$vanishes.

The vector mesons on the meson side of the decay chain can be transversely and longitudinally polarized. We define the corresponding helicity fractions by

$$
\mathcal{F}_{L}=\left|\hat{H}_{\frac{1}{2} 0}\right|^{2}+\left|\hat{H}_{-\frac{1}{2} 0}\right|^{2} \quad \mathcal{F}_{T}=\left|\hat{H}_{\frac{1}{2} 1}\right|^{2}+\left|\hat{H}_{-\frac{1}{2}-1}\right|^{2} .
$$

For the meson-side decay $1^{-} \rightarrow 0^{-}+0^{-}$one obtains the angular decay distribution

$$
W\left(\theta_{V}\right)=\left(\frac{3}{2} \cos ^{2} \theta_{V} \mathcal{F}_{L}+\frac{3}{4} \sin ^{2} \theta_{V} \mathcal{F}_{T}\right) .
$$

The longitudinal polarization of the daughter baryon $\Sigma_{c}^{++}$depends on the polar emission angle $\theta_{V}$ via

$$
P_{B_{2}}\left(\cos \theta_{V}\right)=P_{\Sigma_{c}^{++}}\left(\cos \theta_{V}\right)=\frac{\frac{3}{4} \sin ^{2} \theta_{V}\left(\left|H_{\frac{1}{2}}\right|^{2}-\left|H_{-\frac{1}{2}-1}\right|^{2}\right)+\frac{3}{2} \cos ^{2} \theta_{V}\left(\left|H_{\frac{1}{2}}\right|^{2}-\left|H_{-\frac{1}{2}}\right|^{2}\right)}{\frac{3}{4} \sin ^{2} \theta_{V}\left(\left|H_{\frac{1}{2} 1}\right|^{2}+\left|H_{-\frac{1}{2}-1}\right|^{2}\right)+\frac{3}{2} \cos ^{2} \theta_{V}\left(\left|H_{\frac{1}{2}}\right|^{2}+\left|H_{-\frac{1}{2} 0}\right|^{2}\right)} .
$$

When averaged over $\cos \theta_{V}$ (one has to integrate the numerator and denominator separately) one has

$$
\begin{aligned}
P_{B_{2}}=P_{\Sigma_{c}^{++}} & =\left(\left|\hat{H}_{\frac{1}{1} 1}\right|^{2}-\left|\hat{H}_{-\frac{1}{2}-1}\right|^{2}\right)+\left(\left|\hat{H}_{\frac{1}{2} 0}\right|^{2}-\left|\hat{H}_{-\frac{1}{2} 0}\right|^{2}\right) \\
& =\mathcal{F}_{T}^{P}+\mathcal{F}_{L}^{P} .
\end{aligned}
$$

As mentioned before the polarization of the $\Sigma_{c}^{++}$is not measurable in its strong decay. However, the $\Sigma_{c}^{++}$transfers its polarization to the $\Lambda_{c}^{+}$in the (strong) decay $\Sigma_{c}^{++} \rightarrow$ $\Lambda_{c}^{+} \pi^{+}$where the polarization transfer depends on $\cos \theta_{B}$. The average longitudinal polarization of the $\Lambda_{c}^{+}$can be calculated to be (again we average over $\cos \theta_{V}$ )

$$
P_{\Lambda_{c}^{+}}\left(\theta_{B}\right)=P_{\Sigma_{c}^{++}} \cos \theta_{B} .
$$

As in Eq. (26) one has a sign reversal for the $\Xi_{c}^{+}$in the decay chain $\Xi_{c}^{\prime+}+\Xi_{c}^{+}+\gamma$.

(iii) $1 / 2^{+} \rightarrow 3 / 2^{+}+0^{-}$

In Table VI we list the rates and branching fractions for the four decays in this class.

Contrary to the $1 / 2^{+} \rightarrow 1 / 2^{+}+0^{-}$case the baryon side angular decay distribution of $\Sigma_{c}^{++}\left(2520 ; 3 / 2^{+}\right) \rightarrow$ $\Lambda_{c}^{+} \pi^{+}$now shows a $\theta_{B}$ dependence given by

$$
W\left(\theta_{B}\right)=\left(1-\frac{3}{4} \sin ^{2} \theta_{B}\right) ;
$$

i.e., there is a pronounced dip of the angular decay distribution at $\theta_{B}=90^{\circ}$.

In the constituent quark model the vector transition $1 / 2^{+} \rightarrow 3 / 2^{+}$is conserved; i.e., the vector current helicity amplitude $H_{ \pm 1 / 2 t}^{V}=0$ vanishes for the transition $\Xi_{c c}^{++} \rightarrow \Sigma_{c}^{*++}$. This implies that the final $3 / 2^{+}$state has no polarization structure and therefore there is no polarization transfer to the $\Lambda_{c}^{+}$ in the second stage decay $\Sigma_{c}^{++}\left(2520 ; 3 / 2^{+}\right) \rightarrow$ $\Lambda_{c}^{+} \pi^{+}$. The same statement holds true for the other three $1 / 2^{+} \rightarrow 3 / 2^{+}+0^{-}$decays.

(iv) $1 / 2^{+} \rightarrow 3 / 2^{+}+1^{-}$

In Table VII we list the rates, branching fractions and three polarization parameters $\mathcal{F}_{L}^{P}, \mathcal{F}_{T}^{P}$ and $\mathcal{F}_{T}^{P^{\prime}}$ needed to describe the longitudinal polarization of the daughter baryon $P_{B_{2}}$ for the four decays in this class.

The threefold joint angular decay distribution can be obtained from the first row of Eq. (27) by replacing the Wigner $d^{1 / 2}$-function by the corresponding spin $3 / 2$ Wigner $d^{3 / 2}$-function. Again, one has to observe the angular momentum constraint $\left|\lambda_{2}-\lambda_{M}\right| \leq 1 / 2$. The threefold angular decay distribution reads

TABLE VI. Cabibbo-favored factorizing nonleptonic two-body decays of double heavy charm baryons induced by the quark level $c \rightarrow s ; d \rightarrow u$ transitions for the cases $1 / 2^{+} \rightarrow 3 / 2^{+}+0^{-}$.

\begin{tabular}{llcr}
\hline \hline & & $\Gamma\left[10^{-13} \mathrm{GeV}\right]$ & $\mathcal{B}[\%]$ \\
\hline $1 / 2^{+} \rightarrow 3 / 2^{+}+0^{-}$ & $\Xi_{c c}^{++} \rightarrow \Sigma_{c}^{*++}+\bar{K}^{0}$ & 0.06 & 0.25 \\
& $\Xi_{c c}^{++} \rightarrow \Xi_{c}^{*+}+\pi^{+}$ & 0.16 & 0.63 \\
& $\Omega_{c c}^{+} \rightarrow \Xi_{c}^{*+}+\bar{K}^{0}$ & 0.03 & 0.10 \\
& $\Omega_{c c}^{+} \rightarrow \Omega_{c}^{* 0}+\pi^{+}$ & 0.31 & 1.00 \\
\hline \hline
\end{tabular}


TABLE VII. Cabibbo-favored factorizing nonleptonic two-body decays of double heavy charm baryons induced by the quark level $c \rightarrow s ; d \rightarrow u$ transitions for the case $1 / 2^{+} \rightarrow 3 / 2^{+}+1^{-}$.

\begin{tabular}{llccccc}
\hline \hline & & $\Gamma\left[10^{-13} \mathrm{GeV}\right]$ & $\mathcal{B}[\%]$ & $\mathcal{F}_{L}^{P}$ & $\mathcal{F}_{T}^{P}$ & $\mathcal{F}_{T}^{\prime P}$ \\
\hline $1 / 2^{+} \rightarrow 3 / 2^{+}+1^{-}$ & $\Xi_{c c}^{++} \rightarrow \Sigma_{c}^{*++}+\bar{K}^{* 0}$ & 0.42 & 1.62 & -0.01 & -0.10 & -0.31 \\
& $\Xi_{c c}^{++} \rightarrow \Xi_{c}^{*+}+\rho^{+}$ & 1.15 & 4.48 & -0.01 & -0.08 & -0.24 \\
& $\Omega_{c c}^{+} \rightarrow \Xi_{c}^{*+}+\bar{K}^{* 0}$ & 0.21 & 0.67 & -0.01 & -0.10 & -0.30 \\
& $\Omega_{c c}^{+} \rightarrow \Omega_{c}^{* 0}+\rho^{+}$ & 2.23 & 7.11 & -0.01 & -0.08 & -0.24 \\
\hline \hline
\end{tabular}

$$
\begin{aligned}
W\left(\theta_{V}, \theta_{B}, \chi\right)= & \frac{3}{2} \cos ^{2} \theta_{V}\left(1-\frac{3}{4} \sin ^{2} \theta_{B}\right)\left(\left|\hat{H}_{\frac{1}{2} 0}\right|^{2}+\left|\hat{H}_{-\frac{1}{2}}\right|^{2}\right)+\frac{3}{4} \sin ^{2} \theta_{V}\left(\left(1-\frac{3}{4} \sin ^{2} \theta_{B}\right)\left(\left|\hat{H}_{\frac{1}{2} 1}\right|^{2}+\left|\hat{H}_{-\frac{1}{2}-1}\right|^{2}\right)\right. \\
& \left.+\frac{3}{4} \sin ^{2} \theta_{B}\left(\left|\hat{H}_{\frac{3}{2} 1}\right|^{2}+\left|\hat{H}_{-\frac{3}{2}-1}\right|^{2}\right)\right)+\frac{3}{8}\left(\sqrt { \frac { 3 } { 2 } } \operatorname { s i n } 2 \theta _ { V } \operatorname { s i n } 2 \theta _ { B } \left(\cos \chi \operatorname{Re}\left(\hat{H}_{\frac{3}{2} 1} \hat{H}_{\frac{1}{2} 0}^{*}+\hat{H}_{-\frac{3}{2}-1} \hat{H}_{-\frac{1}{2} 0}^{*}\right)\right.\right. \\
& \left.+\sin \chi \operatorname{Im}\left(\hat{H}_{\frac{3}{2} 1} \hat{H}_{\frac{1}{2} 0}^{*}-\hat{H}_{-\frac{3}{2}-1} \hat{H}_{-\frac{1}{2} 0}^{*}\right)\right)+3 \sqrt{3} \sin ^{2} \theta_{V} \sin ^{2} \theta_{B}\left(\cos 2 \chi \operatorname{Re}\left(\hat{H}_{\frac{3}{2} 1} \hat{H}_{-\frac{1}{2}-1}^{*}+\hat{H}_{-\frac{3}{2}-1} \hat{H}_{\frac{1}{2} 1}^{*}\right)\right. \\
& \left.+\sin 2 \chi \operatorname{Im}\left(\hat{H}_{\frac{3}{2} 1} \hat{H}_{-\frac{1}{2}-1}^{*}-\hat{H}_{-\frac{3}{2}-1} \hat{H}_{\frac{1}{2} 1}^{*}\right)\right) .
\end{aligned}
$$

The angular decay distribution can be seen to integrate to $2 \pi$. It is apparent that there is a rich angular structure in the angular decay distribution (34).

In Eq. (34) we have also included the $T$-odd contributions proportional to $\operatorname{Im}\left(\hat{H}_{\frac{3}{2} 1} \hat{H}_{\frac{1}{2} 0}^{*}-\hat{H}_{-\frac{3}{2}-1} \hat{H}_{-\frac{1}{2} 0}^{*}\right)$ and $\operatorname{Im}\left(\hat{H}_{\frac{3}{2} 1} \hat{H}_{-\frac{1}{2}-1}^{*}-\hat{H}_{-\frac{3}{2}-1} \hat{H}_{\frac{1}{2} 1}^{*}\right)$ even though these contributions vanish in our model calculation because our helicity amplitudes are relatively real. The angular coefficients that multiply the $T$-odd contributions can be seen to involve $T$-odd triple products. For example, one has

$\sin 2 \theta_{V} \sin 2 \theta_{B} \sin \chi$

$$
=4\left(\hat{p}_{\Sigma_{c}^{++}} \cdot \hat{p}_{\Lambda_{c}}\right)\left(\hat{p}_{K^{* 0}} \cdot \hat{p}_{k^{-}}\right)\left(\hat{p}_{\Sigma_{c}^{++}} \times \hat{p}_{\Lambda_{c}}\right) \cdot \hat{p}_{k^{-}},
$$

where the hatted three-momenta are normalized to 1 . The $T$ odd can be fed by either final state interactions or by $C P$ violating interactions. It would be interesting to experimentally check on the existence of such triple-product correlations.

We define polarization parameters that describe the angular decay distribution where we also include the numerical values for the parameters for the cascade decay $\Xi_{c c}^{++} \rightarrow \Sigma_{c}^{*++}\left(\rightarrow \Lambda_{c}^{+}+\pi^{+}\right)+\bar{K}^{* 0}\left(\rightarrow K^{-}+\pi^{+}\right)$. One has

$$
\begin{aligned}
\mathcal{F}_{L} & =\left|\hat{H}_{\frac{1}{2}}\right|^{2}+\left|\hat{H}_{-\frac{1}{2} 0}\right|^{2}=0.40, \\
\mathcal{F}_{T} & =\left|\hat{H}_{\frac{1}{2}}\right|^{2}+\left|\hat{H}_{-\frac{1}{2}-1}\right|^{2}=0.16, \\
\mathcal{F}_{T}^{\prime} & =\left|\hat{H}_{\frac{3}{2}}\right|^{2}+\left|\hat{H}_{-\frac{3}{2}-1}\right|^{2}=0.45, \\
\mathcal{F}_{L}^{P} & =\left|\hat{H}_{\frac{1}{2}}\right|^{2}-\left|\hat{H}_{-\frac{1}{2} 0}\right|^{2}=-0.01, \\
\mathcal{F}_{T}^{P} & =\left|\hat{H}_{\frac{1}{2} 1}\right|^{2}-\left|\hat{H}_{-\frac{1}{2}-1}\right|^{2}=-0.10,
\end{aligned}
$$

$$
\begin{aligned}
\mathcal{F}_{T}^{P^{\prime}} & =\left|\hat{H}_{\frac{3}{2} 1}\right|^{2}-\left|\hat{H}_{-\frac{3}{2}-1}\right|^{2}=-0.31, \\
\gamma & =\operatorname{Re}\left(\hat{H}_{\frac{3}{2} 1} \hat{H}_{\frac{1}{2} 0}^{*}+\hat{H}_{-\frac{3}{2}-1} \hat{H}_{-\frac{1}{2} 0}^{*}\right)=0.39, \\
\gamma^{\prime} & =\operatorname{Re}\left(\hat{H}_{\frac{3}{2} 1} \hat{H}_{-\frac{1}{2}-1}^{*}+\hat{H}_{-\frac{3}{2}-1} \hat{H}_{\frac{1}{2} 1}^{*}\right)=0.19 .
\end{aligned}
$$

By integrating over the respective pairs of angles one obtains the single angle decay distributions

$$
\begin{aligned}
\frac{1}{2 \pi} W\left(\theta_{V}\right) & =\frac{3}{2} \cos ^{2} \theta_{V} \mathcal{F}_{L}+\frac{3}{4} \sin ^{2} \theta_{V}\left(\mathcal{F}_{T}+\mathcal{F}_{T}^{\prime}\right) \\
\frac{1}{2 \pi} W\left(\theta_{B}\right) & =\left(1-\frac{3}{4} \sin ^{2} \theta_{B}\right)\left(\mathcal{F}_{L}+\mathcal{F}_{T}\right)+\frac{3}{4} \sin ^{2} \theta_{B} \mathcal{F}_{T}^{\prime} \\
W(\chi) & =1+2 \sqrt{3} \gamma^{\prime} \cos 2 \chi
\end{aligned}
$$

The contribution of the remaining azimuthal asymmetry parameter $\gamma$ can be obtained by folding the angular decay distribution with $\cos \theta_{V} \cos \theta_{B}$. The numerical values of the angular coefficients are listed in Eq. (36).

The polarization transfer to the $\Lambda_{c}$ in the strong decay $\Sigma_{c}^{*++} \rightarrow \Lambda_{c}^{+}+\pi^{+}$is given by

$$
\begin{aligned}
P_{\Lambda_{c}^{+}}\left(\theta_{B}\right)= & \left(\left(\left|\hat{H}_{\frac{1}{2} 0}\right|^{2}-\left|\hat{H}_{-\frac{1}{2}}\right|^{2}+\left|\hat{H}_{\frac{1}{2}}\right|^{2}-\left|\hat{H}_{-\frac{1}{2}-1}\right|^{2}\right)\right. \\
& \times\left(1-\frac{3}{4} \sin ^{2} \theta_{B}\right) \\
& \left.+\left(\left|\hat{H}_{\frac{3}{2}}\right|^{2}-\left|\hat{H}_{-\frac{3}{2}-1}\right|^{2}\right) \frac{3}{4} \sin ^{2} \theta_{B}\right) \cos \theta_{B} .
\end{aligned}
$$

As in Eq. (26) there is a reversal in sign in the polarization transfer to the stage 2 charm baryons $\Xi_{c}^{+}$and $\Omega_{c}^{0}$ in the last 
three decays of Table VII since the stage 2 decays $\Xi_{c}^{*+} \rightarrow$ $\Xi_{c}^{+}+\gamma$ and $\Omega_{c}^{* 0} \rightarrow \Omega_{c}^{* 0}+\gamma$ are $\sim 100 \%$ one-photon decays.

\section{SUMMARY AND CONCLUSION}

We have cataloged all Cabibbo-favored semileptonic and nonleptonic two-body decays of the three double heavy charm baryon states $\Xi_{c c}^{++}, \Xi_{c c}^{+}$and $\Omega_{c c}^{+}$where the nonleptonic two-body decays are into ground state mesons and baryons. For the semileptonic decays we have calculated rates, branching ratios and helicity fractions of the $W_{\text {off-shell }}^{-}$ using transition form factors calculated in our CCQM quark model. For the nonleptonic decays we have analyzed the topology structure of their various two-body decays in terms of the two $W$-emission (external and internal) or tree topologies and the three $W$-exchange topologies. We have identified two groups of decays $\Xi_{c c}^{++} \rightarrow \Sigma_{c}^{++(*)}+$ $\bar{K}^{0(*)}$ and $\Omega_{c c}^{+} \rightarrow \Omega_{c}^{0(*)}+\pi^{+}\left(\rho^{+}\right)$which proceed by $W$ emission alone and are thus theoretically favored since there is no contamination from $W$-exchange contributions. The $W$-exchange contributions to the decays $\Xi_{c c}^{++} \rightarrow$ $\Xi_{c}^{\prime(*)+}+\pi^{+}\left(\rho^{+}\right)$and $\Omega_{c c}^{+} \rightarrow \Xi_{c}^{\prime(*)+}+\bar{K}^{(*) 0}$ vanish in the $S U(3)$ limit as a consequence of the Körner-Pati-Woo theorem. Using again transition amplitudes from our CCQM quark model for the latter decays we have calculated rates, branching ratios and angular coefficients that characterize the angular decay distributions of the onesided or two-sided cascade decays of the above two classes of decays. The angular decay distributions involving the $1 / 2^{+} \rightarrow 1 / 2^{+}$baryon transitions are markedly different from those of the $1 / 2^{+} \rightarrow 3 / 2^{+}$transitions. In particular, in the $1 / 2^{+} \rightarrow 1 / 2^{+} 1^{-}$cascade decay $\Xi_{c c}^{++} \rightarrow$ $\Sigma_{c}^{++}\left(2455 ; 1 / 2^{+}\right)+\bar{K}^{* 0}$ there are no azimuthal correlations between the two planes formed by the second stage decays $\Sigma_{c}^{++}\left(2455 ; 1 / 2^{+}\right) \rightarrow \Lambda_{c}^{+} \pi^{+}$and $\bar{K}^{* 0} \rightarrow$ $K^{-} \pi^{+}$), whereas the two decay planes become azimuthally correlated in the $1 / 2^{+} \rightarrow 3 / 2^{+}+1^{-}$cascade decay
$\Xi_{c c}^{++} \rightarrow \Sigma_{c}^{*++}\left(2520 ; 3 / 2^{+}\right)+\bar{K}^{* 0}$. Another discriminating feature of these two possible decay paths is that there is pronounced dip in the $\cos \theta$ distribution in the latter case.

Any of the two-body nonleptonic decays of the $\Xi_{c c}^{+}$ and $\Omega_{c c}^{+}$listed in Table I could be explored in the search for the two missing double heavy charm baryon states. If one takes the discovery channels of the $\Xi_{c c}^{++}$as a guide the decays $\Xi_{c c}^{+} \rightarrow \Lambda_{c}^{+}\left(\Sigma_{c}^{(*)+}\right)+\bar{K}^{(*) 0}$ and $\Xi_{c c}^{+} \rightarrow \Xi_{c}^{0(\prime, *)}+\pi^{+}\left(\rho^{+}\right)$ would be good candidates for the discovery of the $\Xi_{c c}^{+}$ while the $\Omega_{c c}^{+}$should be searched for in the decays $\Omega_{c c}^{+} \rightarrow \Xi_{c}^{+(1, *)}+\bar{K}^{(*) 0}$ or $\Omega_{c c}^{+} \rightarrow \Omega_{c}^{0(*)}+\pi^{+}\left(\rho^{+}\right)$. In this paper we have provided first predictions for the branching ratios of the decays $\Xi_{c c}^{++} \rightarrow \Sigma_{c}^{++(*)}+\bar{K}^{0(*)}$, $\Xi_{c c}^{++} \rightarrow \Xi_{c}^{+(1, *)}+\pi^{+}\left(\rho^{+}\right), \Omega_{c c}^{+} \rightarrow \Xi_{c}^{+(1, *)}+\bar{K}^{(*) 0}$ and $\Omega_{c c}^{+} \rightarrow$ $\Omega_{c}^{0(*)}+\pi^{+}\left(\rho^{+}\right)$. In a follow-up paper we plan to also calculate the $W$-exchange contribution to the Cabibbofavored nonleptonic double charm baryon decays with predictions for the remaining decays of Table I not treated in this paper. This includes a calculation of the recently observed decay $\Xi_{c c}^{++} \rightarrow \Xi_{c}^{+}+\pi^{+}$[2].

\section{ACKNOWLEDGMENTS}

This work was funded by the Carl Zeiss Foundation under Project "Kepler Center für Astro- und Teilchenphysik: Hochsensitive Nachweistechnik zur Erforschung des unsichtbaren Universums" (Grant No. 0653-2.8/581/2), by "Verbundprojekt 05A2017CRESST-XENON: Direkte Suche nach Dunkler Materie mit XENON1T/nT und CRESST-III. Teilprojekt 1" (Förderkennzeichen 05A17VTA)," by CONICYT (Chile) under Grants No. PIA/Basal FB0821 and by FONDECYT (Chile) under Grant No. 1191103. M. A. I. acknowledges the support from the PRISMA Cluster of Excellence (Mainz Uni.). M. A. I. and J. G. K. thank the HeisenbergLandau Grant for partial support.
[1] R. Aaij et al. (LHCb Collaboration), Phys. Rev. Lett. 119, 112001 (2017).

[2] R. Aaij et al. (LHCb Collaboration), Phys. Rev. Lett. 121, 162002 (2018).

[3] R. Aaij et al. (LHCb Collaboration), Phys. Rev. Lett. 121, 052002 (2018).

[4] J. G. Körner, M. Krämer, and D. Pirjol, Prog. Part. Nucl. Phys. 33, 787 (1994).

[5] A. De Rujula, H. Georgi, and S. L. Glashow, Phys. Rev. D 12, 3589 (1975).

[6] D. Ebert, R. N. Faustov, V. O. Galkin, and A. P. Martynenko, Phys. Rev. D 66, 014008 (2002).
[7] T. Gutsche, M. A. Ivanov, J. G. Körner, and V. E. Lyubovitskij, Phys. Rev. D 96, 054013 (2017).

[8] Y. Zhang, CERN Seminar (2017), https://indico.cern.ch/ event $/ 632400 /$.

[9] N. Mathur and M. Padmanath, Phys. Rev. D 99, 031501(R) (2019).

[10] A. Faessler, T. Gutsche, M. A. Ivanov, J. G. Körner, and V. E. Lyubovitskij, Phys. Lett. B 518, 55 (2001); A. Faessler, T. Gutsche, M. A. Ivanov, J. G. Körner, V.E. Lyubovitskij, D. Nicmorus, and K. Pumsa-ard, Phys. Rev. D 73, 094013 (2006); Phys. Rev. D 80, 034025 (2009); AIP Conf. Proc. 1257, 311 (2010); T. Branz, A. Faessler, 
T. Gutsche, M. A. Ivanov, J. G. Körner, V. E. Lyubovitskij, and B. Oexl, Phys. Rev. D 81, 114036 (2010).

[11] T. Gutsche, M. A. Ivanov, J. G. Körner, V. E. Lyubovitskij, and Z. Tyulemissov, Phys. Rev. D 99, 056013 (2019).

[12] T. Gutsche, M. A. Ivanov, J. G. Körner, and V. E. Lyubovitskij, Particles 2, 339 (2019).

[13] S. Fleck and J. M. Richard, Prog. Theor. Phys. 82, 760 (1989).

[14] V. V. Kiselev, A. K. Likhoded, and A. I. Onishchenko, Phys. Rev. D 60, 014007 (1999); V. V. Kiselev and A. K. Likhoded, Usp. Fiz. Nauk 172, 497 (2002) [Phys. Usp. 45, 455 (2002)].

[15] S. S. Gershtein, V. V. Kiselev, A. K. Likhoded, and A. I. Onishchenko, Phys. Rev. D 62, 054021 (2000); V. V. Kiselev, A. K. Likhoded, and A. I. Onishchenko, Eur. Phys. J. C 16, 461 (2000); V. V. Kiselev, A. K. Likhoded, O. N. Pakhomova, and V. A. Saleev, Phys. Rev. D 66, 034030 (2002); V. V. Kiselev, A. V. Berezhnoy, and A. K. Likhoded, Phys. At. Nucl. 81, 369 (2018) [Yad. Fiz. 81, 356 (2018)].

[16] D. Ebert, R. N. Faustov, V. O. Galkin, and A. P. Martynenko, Phys. Rev. D 70, 014018 (2004); 77, 079903(E) (2008).

[17] C. Albertus, E. Hernandez, J. Nieves, and J. M. VerdeVelasco, Eur. Phys. J. A 32, 183 (2007); 36, 119(E) (2008); E. Hernandez, J. Nieves, and J. M. Verde-Velasco, Phys. Lett. B 663, 234 (2008); C. Albertus, E. Hernandez, and J. Nieves, Phys. Lett. B 683, 21 (2010); C. Albertus, E. Hernández, and J. Nieves, Phys. Lett. B 704, 499 (2011); C. Albertus, E. Hernandez, and J. Nieves, Phys. Rev. D 85, 094035 (2012).

[18] J. M. Flynn and J. Nieves, Phys. Rev. D 76, 017502 (2007); 77, 099901(E) (2008).

[19] C. H. Chang, T. Li, X. Q. Li, and Y. M. Wang, Commun. Theor. Phys. 49, 993 (2008).

[20] W. Roberts and M. Pervin, Int. J. Mod. Phys. A 24, 2401 (2009).

[21] M. Karliner and J. L. Rosner, Phys. Rev. D 90, 094007 (2014); 96, 033004 (2017); Phys. Rev. Lett. 119, 202001 (2017).

[22] Z. H. Guo, Phys. Rev. D 96, 074004 (2017).

[23] L. Y. Xiao, K. L. Wang, Q. F. Lü, X. H. Zhong, and S. L. Zhu, Phys. Rev. D 96, 094005 (2017).

[24] Q. F. Lü, K. L. Wang, L. Y. Xiao, and X. H. Zhong, Phys. Rev. D 96, 114006 (2017).

[25] N. Sharma and R. Dhir, Phys. Rev. D 96, 113006 (2017).

[26] R. Dhir and N. Sharma, Eur. Phys. J. C 78, 743 (2018).

[27] F. S. Yu, H. Y. Jiang, R. H. Li, C. D. Lü, W. Wang, and Z. X. Zhao, Chin. Phys. C 42, 051001 (2018).

[28] W. Wang, Z. P. Xing, and J. Xu, Eur. Phys. J. C 77, 800 (2017).

[29] Y. J. Shi, W. Wang, Y. Xing, and J. Xu, Eur. Phys. J. C 78, 56 (2018).

[30] E. L. Cui, H. X. Chen, W. Chen, X. Liu, and S. L. Zhu, Phys. Rev. D 97, 034018 (2018).
[31] W. Wang, F. S. Yu, and Z. X. Zhao, Eur. Phys. J. C 77, 781 (2017).

[32] X. H. Hu, Y. L. Shen, W. Wang, and Z. X. Zhao, Chin. Phys. C 42, 123102 (2018).

[33] H. X. Chen, Q. Mao, W. Chen, X. Liu, and S. L. Zhu, Phys. Rev. D 96, 031501(R) (2017); 96, 119902(E) (2017); H. S. Li, L. Meng, Z. W. Liu, and S. L. Zhu, Phys. Rev. D 96, 076011 (2017); Phys. Lett. B 777, 169 (2018); L. Meng, H. S. Li, Z. W. Liu, and S. L. Zhu, Eur. Phys. J. C 77, 869 (2017); Z. X. Zhao, Eur. Phys. J. C 78, 756 (2018); R. H. Li, C. D. Lü, W. Wang, F. S. Yu, and Z. T. Zou, Phys. Lett. B 767, 232 (2017); Z. P. Xing and Z. X. Zhao, Phys. Rev. D 98, 056002 (2018).

[34] Y. J. Shi, Y. Xing, and Z. X. Zhao, Eur. Phys. J. C 79, 501 (2019); Y. J. Shi, W. Wang, and Z.X. Zhao, arXiv: 1902.01092; Q. X. Yu, J. M. Dias, W. H. Liang, and E. Oset, arXiv:1909.13449; X. H. Hu and Y. J. Shi, arXiv: 1910.07909.

[35] T. Branz, A. Faessler, T. Gutsche, M. A. Ivanov, J. G. Körner, and V. E. Lyubovitskij, Phys. Rev. D 81, 034010 (2010).

[36] M. Tanabashi et al., Phys. Rev. D 98, 030001 (2018).

[37] A. K. Leibovich, Z. Ligeti, I. W. Stewart, and M. B. Wise, Phys. Lett. B 586, 337 (2004).

[38] G. Buchalla, A. J. Buras, and M. E. Lautenbacher, Rev. Mod. Phys. 68, 1125 (1996).

[39] J. G. Körner, Nucl. Phys. B25, 282 (1971).

[40] J. C. Pati and C. H. Woo, Phys. Rev. D 3, 2920 (1971).

[41] J. G. Körner, G. Kramer, and J. Willrodt, Z. Phys. C 2, 117 (1979).

[42] J. G. Körner and M. Krämer, Z. Phys. C 55, 659 (1992).

[43] T. Uppal, R. C. Verma, and M. P. Khanna, Phys. Rev. D 49, 3417 (1994).

[44] Fayyazuddin and Riazuddin, Phys. Rev. D 55, 255 (1997); 56, 531(E) (1997).

[45] M. A. Ivanov, J. G. Körner, V. E. Lyubovitskij, and A. G. Rusetsky, Phys. Rev. D 57, 5632 (1998); Mod. Phys. Lett. A 13, 181 (1998).

[46] T. Gutsche, M. A. Ivanov, J. G. Körner, V. E. Lyubovitskij, and P. Santorelli, Phys. Rev. D 88, 114018 (2013).

[47] T. Gutsche, M. A. Ivanov, J. G. Körner, V. E. Lyubovitskij, V. V. Lyubushkin, and P. Santorelli, Phys. Rev. D 96, 013003 (2017).

[48] T. Gutsche, M. A. Ivanov, J. G. Korner, V. E. Lyubovitskij, and P. Santorelli, Phys. Rev. D 93, 034008 (2016).

[49] S. Dubnicka, A. Z. Dubnickova, M. A. Ivanov, and A. Liptaj, Phys. Rev. D 87, 074021 (2013).

[50] M. A. Ivanov, V. E. Lyubovitskij, J. G. Korner, and P. Kroll, Phys. Rev. D 56, 348 (1997).

[51] A. Salam, Nuovo Cimento 25, 224 (1962).

[52] S. Weinberg, Phys. Rev. 130, 776 (1963).

[53] B. König, J. G. Körner, and M. Krämer, Phys. Rev. D 49, 2363 (1994). 